\title{
Cosmic Dark Radiation and Neutrinos
}

\author{
Maria Archidiacono, ${ }^{1}$ Elena Giusarma, ${ }^{2}$ Steen Hannestad, ${ }^{1}$ and Olga Mena ${ }^{2}$ \\ ${ }^{1}$ Department of Physics and Astronomy, University of Aarhus, DK-8000 Aarhus C, Denmark \\ ${ }^{2}$ IFIC, Universidad de Valencia-CSIC, 46071 Valencia, Spain \\ Correspondence should be addressed to Maria Archidiacono; archi@phys.au.dk
}

Received 28 June 2013; Accepted 18 October 2013

Academic Editor: Elisa Bernardini

Copyright (C) 2013 Maria Archidiacono et al. This is an open access article distributed under the Creative Commons Attribution License, which permits unrestricted use, distribution, and reproduction in any medium, provided the original work is properly cited.

\begin{abstract}
New measurements of the cosmic microwave background (CMB) by the Planck mission have greatly increased our knowledge about the universe. Dark radiation, a weakly interacting component of radiation, is one of the important ingredients in our cosmological model which is testable by Planck and other observational probes. At the moment, the possible existence of dark radiation is an unsolved question. For instance, the discrepancy between the value of the Hubble constant, $H_{0}$, inferred from the Planck data and local measurements of $H_{0}$ can to some extent be alleviated by enlarging the minimal $\Lambda \mathrm{CDM}$ model to include additional relativistic degrees of freedom. From a fundamental physics point of view, dark radiation is no less interesting. Indeed, it could well be one of the most accessible windows to physics beyond the standard model, for example, sterile neutrinos. Here, we review the most recent cosmological results including a complete investigation of the dark radiation sector in order to provide an overview of models that are still compatible with new cosmological observations. Furthermore, we update the cosmological constraints on neutrino physics and dark radiation properties focusing on tensions between data sets and degeneracies among parameters that can degrade our information or mimic the existence of extra species.
\end{abstract}

\section{Introduction}

The connection between cosmological observations and neutrino physics is one of the most interesting and hot topics in astroparticle physics.

Earth-based experiments have demonstrated that neutrinos oscillate and therefore have mass (see, e.g., [1] for a recent treatment). However, oscillation experiments are not sensitive to the absolute neutrino mass scale, only the squared mass differences, $\Delta m^{2}$. Furthermore, the sign is known for only one of the two mass differences, namely, $\Delta m_{12}^{2}$, because of matter effects in the Sun. $\Delta m_{23}^{2}$ is currently only measured via vacuum oscillations which depends only on $\left|\Delta m_{23}^{2}\right|$. Even for standard model neutrinos, there are therefore important unresolved questions which have a significant impact on cosmology. Not only is the absolute mass scale not known, but the hierarchy between masses is also unknown. In any case, the two measured mass squared differences imply that at least two neutrinos are very nonrelativistic today (see, e.g., [2] for a recent overview).
Unlike neutrino oscillation experiments, cosmology probes the sum of the neutrino masses (see, e.g., $[3,4]$ ) because it is sensitive primarily to the current neutrino contribution to the matter density. At the moment, cosmology provides a stronger bound on the neutrino mass than laboratory bounds from, for example, beta decay, although the KATRIN experiment is set to improve the sensitivity to $\sum m_{\nu}$ to about $0.6 \mathrm{eV}$ [5].

The tightest $95 \%$ c.l. upper limits to date are $\sum m_{\nu}<$ $0.15 \mathrm{eV}$ [6] and $\sum m_{v}<0.23 \mathrm{eV}$ [7] from different combinations of data sets and different analyses. This astounding accuracy is possible because neutrinos leave key signatures through their free-streaming nature in several cosmological data sets: the temperature-anisotropy power spectrum of the Cosmic Microwave Background (see Section 1.1) and the power spectrum of matter fluctuations, which is one of the basic products of galaxy redshift surveys (see [8]). However, it should be stressed that cosmological constraints are highly model-dependent and, following the Bayesian method, theoretical assumptions have a strong impact on the results and can lead to erroneous conclusions. For instance 
in $[9,10]$, the assumption about spatial flatness is relaxed, testing therefore the impact of a nonzero curvature in the neutrino mass bound. It is also well known that the bound on the neutrino mass is sensitive to assumptions about the dark energy equation of state [11].

In the standard model, there are exactly three neutrino mass eigenstates, $\left(v_{1} v_{2}, v_{3}\right)$, corresponding to the three flavor eigenstates $\left(v_{e}, v_{\mu}, v_{\tau}\right)$ of the weak interaction.

This has been confirmed by precision electroweak measurements at the $Z^{0}$-resonance by the LEP experiment. The invisible decay width of $Z_{0}$ corresponds to $N_{v}=2.9840 \pm$ 0.0082 [12], consistent within $\sim 2 \sigma$ with the known three families of the SM.

In cosmology, the energy density contribution of one $\left(N_{\text {eff }}=1\right)$ fully thermalised neutrino plus antineutrino below the $e^{+} e^{-}$annihilation scale of $T \sim 0.2 \mathrm{MeV}$ is at the lowest order given by $\rho_{\nu}=(7 / 8)(4 / 11)^{4 / 3} \rho_{\gamma}$. However, a more precise calculation which takes into account finite temperature effects on the photon propagator and incomplete neutrino decoupling during $e^{+} e^{-}$annihilation leads to a standard model prediction of $N_{\text {eff }}=3.046$ (see, e.g., [13]). This is not because there is a noninteger number of neutrino species but simply comes from the definition of $N_{\text {eff }}$.

In the last few years, the WMAP satellite as well as the high multipole CMB experiments Atacama Cosmology Telescope (ACT) and South Pole Telescope (SPT) provided some hints for a nonstandard value of the effective number of relativistic degrees of freedom $N_{\text {eff }}$, pointing towards the existence of an extra dark component of the radiation content of the Universe, coined dark radiation.

A variation in $N_{\text {eff }}$ affects both the amplitude and the shape of the Cosmic Microwave Background temperature anisotropy power spectrum (see Section 1.1). Nevertheless, the new data releases of these two experiments (see [14] for ACT and [15] for SPT) seem to disagree in their conclusions on this topic $[16,17]$ : in combination with data from the last data release of the Wilkinson Microwave Anisotropy Probe satellite (WMAP 9 year), SPT data lead to an evidence of an extra dark radiation component $\left(N_{\text {eff }}=3.93 \pm 0.68\right.$ at $68 \%$ c.l.), while ACT data prefer a standard value of $N_{\text {eff }}$ $\left(N_{\text {eff }}=2.74 \pm 0.47\right.$ at $68 \%$ c.l. $)$. The inclusion of external data sets (Baryonic Acoustic Oscillation [18-22] and Hubble Space Telescope measurements [23]) partially reconciles the two experiments in the framework of a $\Lambda$ CDM model with additional relativistic species.

The recently released Planck data have strongly confirmed the standard $\Lambda$ CDM model. The results have provided the most precise constraints ever on the six "vanilla" cosmological parameters [24] by measuring the Cosmic Microwave Background temperature power spectrum up to the seventh acoustic peak [24] with nine frequency channels (100, 143, and $217 \mathrm{GHz}$ are the three frequency channels involved in the cosmological analysis). Concerning dark radiation, Planck results point towards a standard value of $N_{\text {eff }}\left(N_{\text {eff }}=\right.$ $3.36_{-0.64}^{+0.68}$ at $95 \%$ c.l. using Planck data combined with WMAP 9 year polarization measurements and high multipole CMB experiments, both ACT and SPT). However, the $\sim 2.5 \sigma$ tension among Planck and HST measurements of the Hubble constant value can be solved, for instance, by extending the $\Lambda C D M$ model to account for a nonvanishing $\Delta N_{\text {eff }}\left(N_{\text {eff }}=\right.$ $3.62_{-0.48}^{+0.50}$ at $95 \%$ c.l. using Planck $+\mathrm{WP}+$ highL plus a prior on the Hubble constant from the Hubble Space Telescope measurements [23]).

In this review, after explaining the effects of $N_{\text {eff }}$ on CMB power spectrum (Section 1.1), in Section 1.2, we list the different dark radiation models with their state of art constraints on the effective number of relativistic degrees of freedom. Section 2 illustrates the method and the data sets we use here in order to constrain the neutrino parameters we are interested in (number of species and masses). The results of our analyses are reported in Section 3. In Section 4, we present a forecast of the Euclid results on the neutrino number and mass. Finally in Section 5, we discuss our conclusions in light of the former considerations.

1.1. $N_{\text {eff }}$ Effects on Cosmological Observables. The total radiation content of the Universe below the $e^{+} e^{-}$annihilation temperature can be parameterized as follows:

$$
\varrho_{r}=\left[1+\frac{7}{8}\left(\frac{4}{11}\right)^{4 / 3} N_{\mathrm{eff}}\right] \varrho_{\gamma},
$$

where $\rho_{\gamma}$ is the energy density of photons, $7 / 8$ is the multiplying factor for each fermionic degree of freedom, and $(4 / 11)^{1 / 3}$ is the photon neutrino temperature ratio. Finally, the parameter $N_{\text {eff }}$ can account for neutrinos and for any extra relativistic degrees of freedom; namely, are particles are still relativistic at decoupling as follows:

$$
N_{\text {eff }}=3.046+\Delta N_{\text {eff }}
$$

Varying $N_{\text {eff }}$ changes the time of the matter radiation equivalence: a higher radiation content due to the presence of additional relativistic species leads to a delay in $z_{\text {eq }}$ as follows:

$$
1+z_{\mathrm{eq}}=\frac{\Omega_{m}}{\Omega_{r}}=\frac{\Omega_{m} h^{2}}{\Omega_{\gamma} h^{2}} \frac{1}{\left(1+0.2271 N_{\mathrm{eff}}\right)},
$$

where $\Omega_{m}$ is the matter density, $\Omega_{r}$ is the radiation density, $\Omega_{\gamma}$ is the photon density, $h$ is defined as $H_{0}=100 h \mathrm{~km} / \mathrm{s} / \mathrm{Mpc}$, and in the last equality we have used equation (1). As a consequence at the time of decoupling, radiation is still a subdominant component and the gravitational potential is still slowly decreasing. This shows up as an enhancement of the early Integrated Sachs Wolfe (ISW) effect that increases the CMB perturbation peaks at $\ell \sim 200$, that is, around the first acoustic peak. This effect is demonstrated in Figure 1.

In [25], the authors stress that the most important effect of changing $N_{\text {eff }}$ is located at high $\ell>600$ and is not related to the early ISW effect. Indeed, the main effect related to a variation of the number of relativistic species at decoupling is that it alters the expansion rate, $H$, around the epoch of last scattering. The extra dark radiation component, arising from a value of $N_{\text {eff }}$ greater than the standard 3.046, contributes to the expansion rate via its energy density $\Omega_{\mathrm{DR}}$ as follows:

$$
\frac{H^{2}}{H_{0}^{2}}=\frac{\Omega_{m}}{a^{3}}+\Omega_{\Lambda}+\frac{\Omega_{\gamma}}{a^{4}}+\frac{\Omega_{v}}{a^{4}}+\frac{\Omega_{\mathrm{DR}}}{a^{4}} .
$$




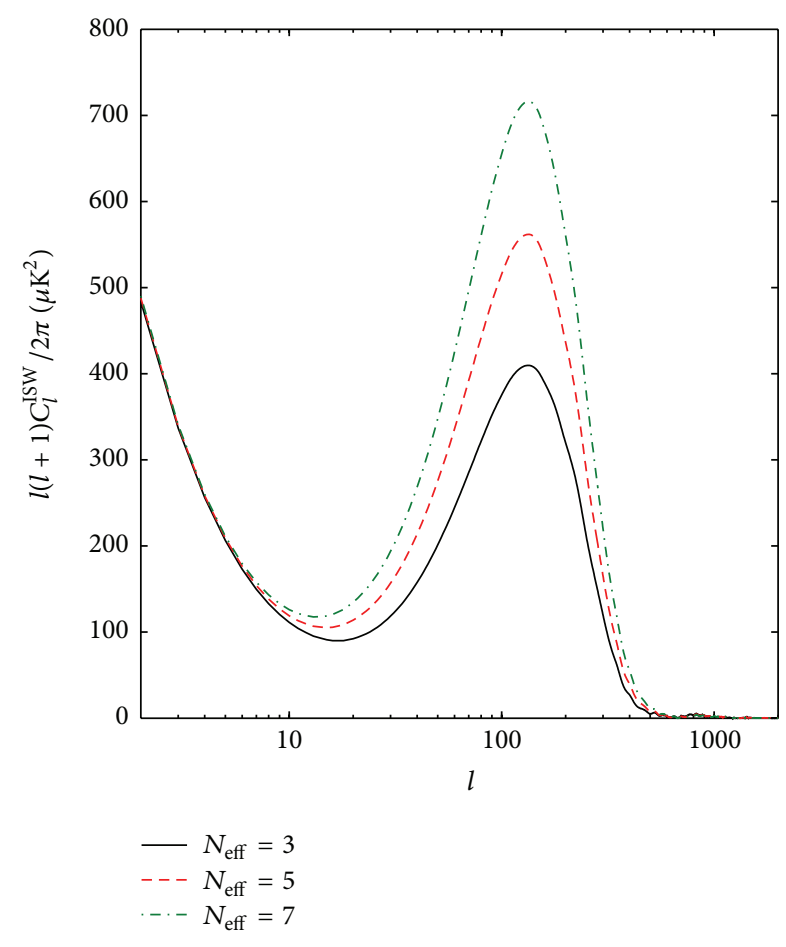

FIGURE 1: ISW contribution to the CMB temperature power spectrum. The raise at $\ell<30$ is due to the late Integrated Sachs Wolfe, while the peak around $\ell \sim 200$ is the early Integrated Sachs Wolfe effect. The cosmological model is the $\Lambda \mathrm{CDM}$ with $N_{\text {eff }}$ being equal to 3 (black solid line), 5 (red dashed line), and 7 (green dot-dashed line).

If $N_{\text {eff }}$ increases, $H$ increases as well. Furthermore, the delay in matter radiation equality, which causes the early ISW, also modifies the baryon to photon density ratio as follows:

$$
R_{\mathrm{eq}}=\left.\frac{3 \rho_{b}}{4 \rho_{\gamma}}\right|_{a_{\mathrm{eq}}}
$$

and therefore the sound speed

$$
c_{s}=\frac{1}{\sqrt{3\left(1+R_{\mathrm{eq}}\right)}} .
$$

The size of the comoving sound horizon $r_{s}$ is given by

$$
r_{s}=\int_{0}^{\tau^{\prime}} d \tau c_{s}(\tau)=\int_{0}^{a} \frac{d a}{a^{2} H} c_{s}(a)
$$

and is proportional to the inverse of the expansion rate $r_{s} \propto$ $1 / H$, when $N_{\text {eff }}$ increases, $r_{s}$ decreases. The consequence is a reduction in the angular scale of the acoustic peaks $\theta_{s}=$ $r_{s} / D_{A}$, where $D_{A}$ is the angular diameter distance. The overall effect on the CMB power spectrum is a horizontal shift of the peak positions towards higher multipoles. In Figure 2(b), the total temperature power spectrum is corrected for this effect: the $\ell$ axis is rescaled by a constant factor $\theta_{s}\left(N_{\text {eff }}\right) / \theta_{s}\left(N_{\text {eff }}=3\right)$ in order to account for the peak shift due to the increase in $N_{\text {eff. }}$. Effectively, it amounts to having the same sound horizon for all the models. Considering that $\theta_{s}$ is the most wellconstrained quantity by CMB measures, this is the dominant effect of a varying $N_{\text {eff }}$ on the CMB power spectrum.

Besides the horizontal shift, there is also a vertical shift that affects the amplitude of the peaks at high multipoles where the ISW effect is negligible. Comparing Figure 2 with Figure 1, one can also notice that for a larger value of $N_{\text {eff }}$ the early ISW causes an increase of power on the first and the second peaks, while the same variation in $N_{\text {eff }}$ turns out in a reduction of power in the peaks at higher multipoles. This vertical shift is related to the Silk damping effect. The decoupling of baryon-photon interactions is not instantaneous but rather an extended process. This leads to diffusion damping of oscillations in the plasma, an effect known as Silk damping. If decoupling starts at $\tau_{d}$ and ends at $\tau_{l s}$, during $\Delta \tau$ the radiation free streams on scale $\lambda_{d}=$ $(\lambda \Delta \tau)^{1 / 2}$ where $\lambda$ is the photon mean free path and $\lambda_{d}$ is shorter than the thickness of the last scattering surface. As a consequence, temperature fluctuations on scales smaller than $\lambda_{D}$ are damped, because on such scales photons can spread freely both from overdensities and from underdensities. The damping factor is $\exp \left[-\left(2 r_{d} / \lambda_{d}\right)\right]$ where $r_{d}$ is the mean square diffusion distance at recombination. An approximated expression of $r_{d}$ is given by [25]

$$
r_{d}^{2}=(2 \pi)^{2} \int_{0}^{a_{l s}} \frac{d a}{a^{3} \sigma_{T} n_{e} H}\left[\frac{R^{2}+(6 / 15)(1+R)}{6\left(1+R^{2}\right)}\right],
$$

where $\sigma_{T}$ is the Thompson cross section, $n_{e}$ is the number density of free electrons, $a_{l s}$ is the scale factor at recombination, and the factor in square brackets is related to polarization [26]. This diffusion process becomes more and more effective approaching the last scattering, so we can consider $a$ constant and thus obtain $r_{d} \propto 1 / \sqrt{H}$. Recalling the dependence $r_{s} \propto 1 / H$ and the fact that $\theta_{s}=r_{s} / D_{A}$ is fixed by $\mathrm{CMB}$ observations, we can infer $D_{A} \propto 1 / H$. The result is that the damping angular scale $\theta_{d}=r_{d} / D_{A}$ is proportional to the square root of the expansion rate $\theta_{d} \propto \sqrt{H}$ and consequently it increases with the number of relativistic species. The effect on the $\mathrm{CMB}$ power spectrum can be seen in Figures 2(c) and 2(d), where, in addition to the $\ell$ rescaling, we have subtracted the ISW power spectrum of Figure 1 in the Figure 2(c), while in the Figure 2(d) we have taken into account the total ISW contribution (both the autocorrelation and the cross correlation). This damping effect shows up as a suppression of the peaks and a smearing of the oscillations that intensifies at higher multipoles.

It is important to stress that all these effects (on the redshift of equivalence, on the size of the sound horizon at recombination, and on the damping tail) can be compensated by varying other cosmological parameters [27]. For instance the damping scale is affected by the helium fraction as well as by the effective number of relativistic degrees of freedom: $r_{d} \propto\left(1-Y_{\mathrm{he}}\right)^{-0.5}$ [25]. Therefore, at the level of the damping in the power spectrum, a larger value of $N_{\text {eff }}$ can be mimicked by a lower value of $Y_{\text {he }}$ (see Figure 5, Section 3.1). The redshift of the equivalence $z_{\text {eq }}$ can be kept fixed by increasing the cold dark matter density while increasing $N_{\text {eff. Finally an }}$ 


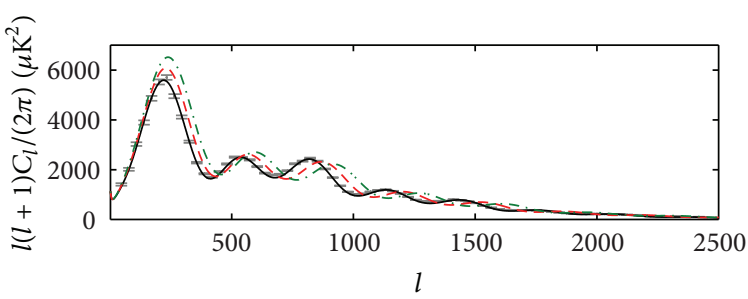

(a)

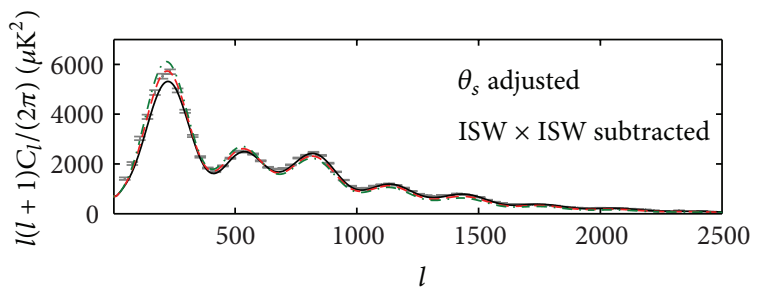

$\begin{aligned} &- N_{\text {eff }}=3 \\ &--- N_{\text {eff }}=5 \\ &-\cdot-N_{\text {eff }}=7\end{aligned}$

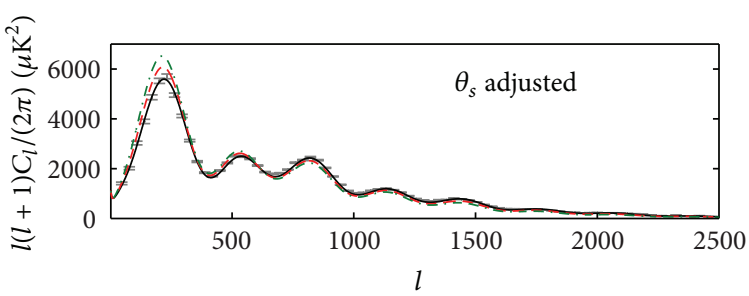

(b)

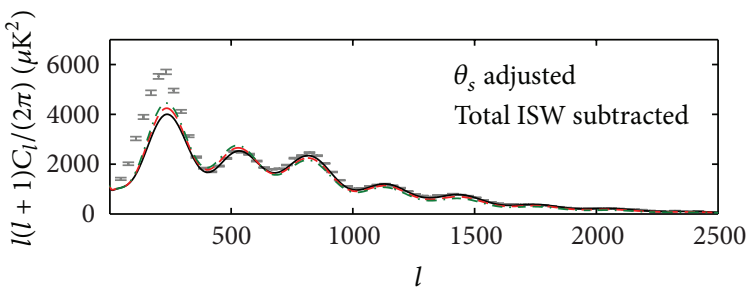

$$
\begin{aligned}
& -N_{\text {eff }}=3 \\
& --N_{\text {eff }}=5 \\
& --N_{\text {eff }}=7
\end{aligned}
$$

(c)

(d)

FIGURE 2: CMB temperature power spectrum. The model and the legend are the same as in Figure 1, the grey error bars correspond to Planck data. (a) The total CMB temperature power spectrum. (b) The $\ell$ axis has been rescaled by a factor $\theta_{s}\left(N_{\text {eff }}\right) / \theta_{s}\left(N_{\text {eff }}=3\right)$. (c) The total ISW $\times$ ISW contribution has been subtracted. (d) The total ISW (both the autocorrelation and the cross correlation) contribution have been subtracted.

open Universe with a nonzero curvature can reproduce the same peak shifting of a larger number of relativistic degrees of freedom. All these degeneracies increase the uncertainty on the results and degrade the constraint on $N_{\text {eff }}$.

The only effect that cannot be mimicked by other cosmological parameters is the neutrino anisotropic stress. The anisotropic stress arises from the quadrupole moment of the cosmic neutrino background temperature distribution and it alters the gravitational potentials [28, 29]. The effect on the CMB power spectrum is located at scales that cross the horizon before the matter-radiation equivalence and it consists of an increase in power by a factor $5 /\left(1+(4 / 15) f_{v}\right)$ [30], where $f_{\nu}$ is the fraction of radiation density contributed by free-streaming particles.

1.2. Dark Radiation Models. A number of theoretical physics models could explain a contribution to the extra dark radiation component of the universe, that is, to $\Delta N_{\text {eff. }}$.

A particularly simple model, based on neutrino oscillation Short BaseLine (SBL) physics results, contains sterile neutrinos. Sterile neutrinos are right-handed fermions which do not interact via any of the fundamental standard model interactions and therefore their number is not determined by any fundamental symmetry in nature. Originally, models with one additional massive mainly sterile neutrino $v_{4}$, with a mass splitting $\Delta m_{14}^{2}$, that is, the so called $(3+1)$ models, were introduced to explain LSND (Large Scintillator Neutrino Detector) [31] SBL antineutrino data by means of neutrino oscillations [32, 33]. A much better fit to both appearance and disappearance data was in principle provided by the $(3+2)$ models [34] in which there are two mostly sterile neutrino mass states $v_{4}$ and $v_{5}$ with mass splittings in the range $0.1 \mathrm{eV}^{2}<\left|\Delta m_{14}^{2}\right|,\left|\Delta m_{15}^{2}\right|<10 \mathrm{eV}^{2}$. In the two sterile neutrino scenarios we can distinguish two possibilities, one in which both mass splittings are positive, named as $3+$ 2 , and one in which one of them is negative, named as $1+3+1$ [35]. Recent MiniBooNE antineutrino data are consistent with oscillations in the $0.1 \mathrm{eV}^{2}<\left|\Delta m_{14}^{2}\right|,\left|\Delta m_{15}^{2}\right|<$ $10 \mathrm{eV}^{2}$, showing some overlapping with LSND results [36]. The running in the neutrino mode also shows an excess at low energy. However, the former excess seems to be not compatible with a simple two-neutrino oscillation formalism [36]. A recent global fit to long baseline, short baseline, solar, and atmospheric neutrino oscillation data [37] has shown that in the $3+1$ and $3+2$ sterile neutrino schemes there is some tension in the combined fit to appearance and disappearance data. This tension is alleviated in the $1+3+1$ sterile neutrino model case with a $P$ value of $0.2 \%$. These results are in good agreement with those presented in [38], which also considered the $3+3$ sterile neutrino models with three active and three sterile neutrinos. They conclude that $3+3$ neutrino models yield a compatibility of $90 \%$ among all short baseline data sets highly superior to those obtained in models with either one or two sterile neutrino species. The existence of this extra sterile neutrinos states can be in tension with Big Bang Nucleosynthesis (see Section 2.2). However, the extra neutrino species may not necessarily be fully thermalised in the early universe. Even though the masses and mixing angles necessary to explain oscillation data would seem to indicate full thermalisation, the presence of, for example, a lepton asymmetry can block sterile neutrino 
production and lead to a significantly lower final abundance, making the model compatible with BBN bounds, see [39-45].

However, an extra radiation component may arise from many other physical mechanisms, as, for instance, QCD thermal axions or extended dark sectors with additional relativistic degrees of freedom. Both possibilities are closely related to minimal extensions to the standard model of elementary particles. Cosmological data provide a unique opportunity to place limits on any model containing new light species, see [46].

We first briefly review the hadronic axion model $[47,48]$ since these hypothetical particles provide the most elegant and promising solution to the strong CP problem. Quantum Chromodynamics (QCD) respects CP symmetry, despite the existence of a natural, four-dimensional Lorentz and gauge invariant operator which violates $\mathrm{CP}$. The presence of this $\mathrm{CP}$ violating-term will induce a nonvanishing neutron dipole moment, $d_{n}$. However, the experimental bound on the dipole moment $\left|d_{n}\right|<3 \times 10^{-26} e \mathrm{~cm}$ [49] would require a negligible CP violation contribution. Peccei and Quinn $[50,51]$ introduced a new global $U(1)_{P Q}$ symmetry, which is spontaneously broken at a scale $f_{a}$, generating a new spinless particle, the axion. The axion mass is inversely proportional to the axion decay constant $f_{a}$ which is the parameter controlling the interaction strength with the standard model plasma and therefore the degree of thermalisation in the early universe. The interaction Lagrangian is proportional to $1 / f_{a}$ and high mass axions therefore have a stronger coupling to the standard model and thermalise more easily. Axions produced via thermal processes in the early Universe provide a possible (sub)dominant hot dark matter candidate, similar, but not exactly equivalent to, neutrino hot dark matter. High mass axions are disfavored by cosmological data, with the specific numbers depending on the model and data sets used (see, e.g., [52-55]). Even though moderate mass axions can still provide a contribution to the energy density we also stress that just as for neutrino hot dark matter it cannot be mapped exactly to a change in $N_{\text {eff }}$.

Generally, any model with a dark sector with relativistic degrees of freedom that eventually decouple from the standard model sector will also contribute to $N_{\text {eff. }}$ Examples are the asymmetric dark matter scenarios (see, e.g., $[56,57]$ and references therein) or extended weakly interacting massive particle models (see the recent work presented in [58-60]). We will review here the expressions from [57], in which the authors include both light $\left(g_{\ell}\right)$ and heavy $\left(g_{h}\right)$ relativistic degrees of freedom at the temperature of decoupling $T_{D}$ from the standard model. For high decoupling temperature, $T_{D}>$ $\mathrm{MeV}$, the dark sector contribution to $N_{\text {eff }}$ is read [57] as

$$
\Delta N_{\mathrm{eff}}=\frac{13.56}{g_{\star S}\left(T_{D}\right)^{4 / 3}} \frac{\left(g_{\ell}+g_{h}\right)^{4 / 3}}{g_{\ell}^{1 / 3}},
$$

where $g_{\star S}\left(T_{D}\right)$ refers to the effective number of entropy degrees of freedom at the dark sector decoupling temperature. If the dark sector decouples at lower temperatures $\left(T_{D}<\right.$ $\mathrm{MeV}$ ), there are two possibilities for the couplings of the dark sector with the standard model: either the dark sector couples to the electromagnetic plasma or it couples to neutrinos. In this former case,

$$
\begin{aligned}
N_{\mathrm{eff}}= & \left(3+\frac{4}{7} \frac{\left(g_{h}+g_{\ell}\right)^{4 / 3}}{g_{\ell}^{1 / 3}}\right) \\
& \times\left(\frac{3 \times 7 / 4+g_{H}+g_{h}+g_{\ell}}{3 \times 7 / 4+g_{h}+g_{\ell}}\right)^{4 / 3},
\end{aligned}
$$

having $g_{H}$ the number of degrees of freedom that become nonrelativistic between typical $\mathrm{BBN}$ temperatures and $T_{D}$. The authors of [57] have shown that the cosmological constraints on $N_{\text {eff }}$ can be translated into the required heavy degrees of freedom heating the light dark sector plasma $g_{h}$ as a function of the dark sector decoupling temperature $T_{D}$ for a fixed value of $g_{\ell}$. Recent Planck data [24], combined with measurements of the Hubble constant $H_{0}$ from the Hubble Space Telescope (HST), low multipole polarization measurements from the Wilkinson Microwave Anisotropy Probe (WMAP) 9 year data release [61], and high multipole $\mathrm{CMB}$ data from both the Atacama Cosmology Telescope (ACT) [14] and the South Pole Telescope (SPT) [15, 62], provide that the constraint $N_{\text {eff }}$ is $3.62_{-0.48}^{+0.50}$ at $95 \%$ c.l. Using this constraint, the authors of [63] have found that having extra heavy degrees of freedom in the dark sector for low decoupling temperatures is highly disfavored.

Another aspect of dark radiation is that it could interact with the dark matter sector. In asymmetric dark matter models (see [56]), the dark matter production mechanism resembles to the one in the baryonic sector, with a particleantiparticle asymmetry at high temperatures. The thermally symmetric dark matter component eventually annihilates and decays into dark radiation species. Due to the presence of such an interaction among the dark matter and dark radiation sectors, they behave as a tightly coupled fluid with pressure which will imprint oscillations in the matter power spectrum (as the acoustic oscillations in the photon-baryon fluid before the recombination era). The clustering properties of the dark radiation component may be modified within interacting schemes, and therefore the clustering parameters $c_{\mathrm{eff}}^{2}$ and $c_{\mathrm{vis}}^{2}$ may differ from their standard values for the neutrino case $c_{\text {eff }}^{2}=c_{\text {vis }}^{2}=1 / 3$ (see Section 2.2). In the presence of a dark radiation-dark matter interaction, the complete Euler equation for dark radiation, including the interaction term with dark matter, is read as follows:

$$
\begin{aligned}
\dot{\theta}_{d r}= & 3 k^{2} c_{\mathrm{eff}}^{2}\left(\frac{1}{4} \delta_{d r}-\frac{\dot{a}}{a} \frac{\theta_{d r}}{k^{2}}\right)-\frac{\dot{a}}{a} \theta_{d r} \\
& -\frac{1}{2} k^{2} \pi_{d r}+a n_{d m} \sigma_{d m-d r}\left(\theta_{d m}-\theta_{d r}\right),
\end{aligned}
$$

where the term $a n_{d m} \sigma_{d m-d r}\left(\theta_{d m}-\theta_{d r}\right)$ represents the moment transferred to the dark radiation component and the quantity $a n_{d m} \sigma_{d m-d r}$ gives the scattering rate of dark radiation by dark matter. The authors of [64] have parameterized the coupling between dark radiation and dark matter through a cross section given by

$$
\left\langle\sigma_{d m-d r}|v|\right\rangle \sim Q_{0} m_{d m}
$$


if it is constant, or

$$
\left\langle\sigma_{d m-d \mathrm{r}}|v|\right\rangle \sim \frac{Q_{2}}{a^{2}} m_{d m}
$$

if it is proportional to $T^{2}$, where the parameters $Q_{0}$ and $Q_{2}$ are constants in $\mathrm{cm}^{2} \mathrm{MeV}^{-1}$ units. It has been shown in [57] that the cosmological implications of both constant and $T$-dependent interacting cross sections are very similar. Recent cosmological constraints on generalized interacting dark radiation models have been presented in [65]; here the authors have shown that if the dark radiation and the dark matter sectors interact in nature, the errors on the dark radiation clustering properties largely increase.

\section{Analysis Method}

The parameter space (see Section 2.2) is sampled through a Monte Carlo Markov Chain performed with the publicly available package CosmoMC [66] based on the MetropolisHastings sampling algorithm and on the Gelman Rubin convergence diagnostic. The calculation of the theoretical observables is done through CAMB [67] (Code for Anisotropies in the Microwave Background) software. The code is able to fit any kind of cosmological data with a bayesian statistic; in our case, we focus on the data sets reported in the following section.

2.1. Data Sets. Our basic data set is the Planck temperature power spectrum (both at low $\ell$ and at high $\ell$ ) in combination with the WMAP 9 year polarization data (hereafter WP) and the high multipole CMB data of ACT and SPT (hereafter highL). These data sets are implemented in the analysis following the prescription of the Planck likelihood described in [24]. The additional data sets test the robustness at low redshift of the predictions obtained with $\mathrm{CMB}$ data. These data sets consist of a prior on the Hubble constant from the Hubble Space Telescope measurements [23] (hereafter $H_{0}$ ) and the information on the dark matter clustering from the matter power spectrum extracted from the Data Release 9 (DR9) of the CMASS sample of galaxies [68] from the Baryon Acoustic Spectroscopic Survey (BOSS) [19], part of the program of the Sloan Digital Sky Survey III [69].

2.2. Parameters. In Table 1 , the parameters used in the analyses are listed together with the top-hat priors on them. The six standard parameters of the $\Lambda$ CDM model are: the physical baryon density, $\omega_{b} \equiv \Omega_{b} h^{2}$; the physical cold dark matter density, $\omega_{c} \equiv \Omega_{c} h^{2}$; the angular scale of the sound horizon, $\theta_{s}$; the reionization optical depth, $\tau$; the amplitude of the primordial spectrum at a certain pivot scale, $A_{s}$; and the power law spectral index of primordial density (scalar) perturbations, $n_{s}$.

We include the effective number of relativistic degrees of freedom $N_{\text {eff }}$, and, in addition, our runs also contain one or a combination of the following parameters: the sum of neutrino masses $\sum m_{\gamma}$, the primordial helium fraction $Y_{\text {he }}$, and the neutrino perturbation parameters, namely, the effective sound speed $c_{\text {eff }}^{2}$ and the viscosity parameter $c_{\mathrm{vis}}^{2}$.
TABLE 1: Priors on the cosmological parameters considered in this work. All priors are uniform (top hat) in the given intervals.

\begin{tabular}{lc}
\hline Parameter & Prior \\
\hline$\omega_{b}$ & $0.005 \rightarrow 0.1$ \\
$\omega_{\text {cdm }}$ & $0.001 \rightarrow 0.99$ \\
$\theta_{s}$ & $0.5 \rightarrow 10$ \\
$\tau$ & $0.01 \rightarrow 0.8$ \\
$\ln \left(10^{10} A_{s}\right)$ & $2.7 \rightarrow 4$ \\
$n_{s}$ & $0.9 \rightarrow 1.1$ \\
\hline$A_{L}$ & $0 \rightarrow 5$ \\
$N_{\text {eff }}$ & $0 \rightarrow 7$ \\
$\sum m_{v}[\mathrm{eV}]$ & $0 \rightarrow 7$ \\
$Y_{\text {he }}$ & $0.1 \rightarrow 0.5$ \\
$c_{\text {eff }}^{2}$ & $0 \rightarrow 1$ \\
$c_{\text {vis }}^{2}$ & $0 \rightarrow 1$ \\
\hline
\end{tabular}

Finally we also investigated the impact of a varying lensing amplitude $A_{L}$.

We assume that massive neutrinos are degenerate and share the same mass. Indeed given the present accuracy of CMB measurements, cosmology cannot extract the neutrino mass hierarchy but only the total hot dark matter density. Even if the future measurements of the Euclid survey will achieve an extreme accurate measurement of the neutrino mass $\left(\sigma_{m_{v}} \simeq 0.01 \mathrm{eV}[70]\right)$, the neutrino mass hierarchy would not be pin down.

2.2.1. Primordial Helium Fraction. The primordial helium fraction, $Y_{\text {he }}$, is a probe of the number of relativistic species at the time of Big Bang Nucleosynthesis. As we have seen in Section 1.1, when $N_{\text {eff }}$ increases, the expansion rate increases as well. This means that free neutrons have less time to convert to protons through beta decay before the freeze out and so the final neutron-to-proton ratio is larger. The observable consequence is that the helium fraction is higher.

Measurements of the primordial light element abundances seem consistent with a standard number of relativistic species at the time of BBN at $95 \%$ c.l. $\left(N_{\text {eff }}^{\mathrm{BBN}}=3.80_{-0.70}^{+0.80}\right.$ at $2 \sigma[71])$. This result is also consistent with the $\mathrm{CMB}$ value $N_{\text {eff }}^{\mathrm{CMB}}=3.68_{-0.64}^{+0.68}$ at $95 \%$ c.l. obtained with the combination of data sets Planck + WP + highL. Nevertheless a tension among $N_{\text {eff }}^{\mathrm{BBN}}$ and $N_{\text {eff }}^{\mathrm{CMB}}$ arises if the $H_{0}$ prior is taken into account; indeed in this case $N_{\text {eff }}^{\mathrm{CMB}}=3.62_{-0.48}^{+0.50}$ at $95 \%$ c.l. However the value of $N_{\text {eff }}$ at $\mathrm{BBN}(T \sim 1 \mathrm{MeV})$ and the value measured by $\mathrm{CMB}$ at the last scattering epoch $(T \sim 1 \mathrm{eV})$ may be different because of the unknown physics in the region $1 \mathrm{MeV}<T<$ $1 \mathrm{eV}$ (see [72] for a recent review). Several efforts have been carried out in order to reconcile $N_{\text {eff }}^{\mathrm{BBN}}$ with the existence of extra species: decay of massive particles $(1 \mathrm{MeV}<m<1 \mathrm{eV})$ in additional relativistic species [73, 74], decay of gravitino into axino and axion [75], or neutrino asymmetries [76].

The BBN consistency relation implies that the number of relativistic species present at $\mathrm{BBN}$ is the same as the number measured by $\mathrm{CMB}$ at recombination. In order to impose the $\mathrm{BBN}$ consistency, we use the standard option implemented 
in CosmoMC [77]. This routine calculates $Y_{\mathrm{he}}$ as a function of $N_{\text {eff }}$ and $\Omega_{b} h^{2}$ using a fitting formula obtained with the ParthENoPE code [78].

2.2.2. Lensing Amplitude. Massive neutrinos suppress the growth of dark matter perturbations both through free streaming and through the equivalence delay. As a consequence, the matter power spectrum is damped on scales smaller than the scale of the horizon when neutrinos become nonrelativistic. The accuracy level of Planck allows for a detection of this clustering suppression in the CMB lensing potential, so it is timely to investigate the correlation among $A_{L}$ and neutrino parameters. Planck analysis [24] provides an anomalous value of the lensing amplitude $A_{L}=1.23 \pm 0.11$ ( $68 \%$ c.l., Planck $+\mathrm{WP}+$ highL). This anomaly was already revealed by ACT data $\left(A_{L}=1.70 \pm 0.38\right.$ at $68 \%$ c.l. [14] $)$ even if with a lower precision. On the contrary, the SPT value $\left(A_{L}=0.86_{-0.13}^{+0.15}\right.$ at $68 \%$ c.l. [62]) is consistent with the standard prediction $A_{L}=1$ within $1 \sigma$. Subsequent analyses [79] have confirmed this anomaly and studied the impact on massless $N_{\text {eff. }}$.

Even if a modification of General Relativity cannot be ruled out, this anomaly is most likely a spurious signal related to the bias induced by the combination of data sets belonging to different experiments with different experimental techniques and different analysis methods. However, it is important to account for its effect in order to get unbiased constraints on the sum of neutrino masses, that is correlated with $A_{L}$, as we will see in Section 3.2.

2.2.3. Neutrino Perturbation Parameters. As we have seen in Section 1.2, there is a wide variety of models that can explain an excess in the number of relativistic degrees of freedom at decoupling. In order to distinguish between these models, we introduce the neutrino perturbation parameters, the effective sound speed, and the viscosity parameter, $c_{\mathrm{eff}}^{2}$ and $c_{\mathrm{vis}}^{2}$, respectively $[80,81]$. The reason is that these parameters can characterize the properties of the component that accounts for extra relativistic species.

Following [82, 83], we encode $c_{\mathrm{eff}}^{2}$ and $c_{\mathrm{vis}}^{2}$ in the massless neutrino perturbation equations as follows:

$$
\begin{gathered}
\dot{\delta}_{v}=\frac{\dot{a}}{a}\left(1-3 c_{\mathrm{eff}}^{2}\right)\left(\delta_{v}+3 \frac{\dot{a}}{a} \frac{q_{v}}{k}\right)-k\left(q_{v}+\frac{2}{3 k} \dot{h}\right), \\
\dot{q}_{v}=k c_{\mathrm{eff}}^{2}\left(\delta_{v}+3 \frac{\dot{a}}{a} \frac{q_{v}}{k}\right)-\frac{\dot{a}}{a} q_{v}-\frac{2}{3} k \pi_{v}, \\
\dot{\pi}_{v}=3 c_{\mathrm{vis}}^{2}\left(\frac{2}{5} q_{v}+\frac{8}{15} \sigma\right)-\frac{3}{5} k F_{v, 3}, \\
\frac{2 l+1}{k} \dot{F}_{v, l}-l F_{v, l-1}=-(l+1) F_{v, l+1}, \quad l \geq 3 .
\end{gathered}
$$

Here, the equations are written in the synchronous gauge (the one used in CAMB package [67]), the dot indicates the derivative respect to conformal time $\tau, a$ is the scale factor, $k$ is the wavenumber, $\delta_{\nu}$ is the neutrino density contrast, $q_{\nu}$ is the neutrino velocity perturbation, $\pi_{\nu}$ is the neutrino anisotropic stress, and $F_{\gamma, \ell}$ are higher-order moments of the neutrino distribution function and $\sigma$ is the shear.
The viscosity parameter is related to the clustering properties of particles, because it parameterizes the relationship between velocity/metric shear and anisotropic stress: $c_{\mathrm{vis}}^{2}=$ 0 indicates a perfect fluid with undamped perturbations, while an increased value of $c_{\text {vis }}^{2}$ causes an overdamping of the oscillations. Free streaming particles, such as neutrinos, lead to anisotropies in the Cosmic Neutrino Background that are characterized by $c_{\mathrm{vis}}^{2}=1 / 3$.

When $c_{\text {eff }}^{2}$ decreases, the internal pressure of the dark radiation fluid decreases and its perturbations can grow and start clustering; on the contrary, if $c_{\mathrm{eff}}^{2}$ increases the oscillations are damped. Furthermore, an increase (decrease) in $c_{\mathrm{eff}}^{2}$ leads to an increase (decrease) in the neutrino sound horizon and, as a consequence, also in the scale at which neutrino perturbations affect the dark radiation fluid.

If the additional relativistic species we are dealing with consist of free streaming particles, such as neutrinos, the perturbation parameters would be $c_{\mathrm{eff}}^{2}=c_{\mathrm{vis}}^{2}=1 / 3$.

\section{Results}

In what follows, the results of our analyses are presented. These results cover a wide range of different parameter spaces and they are obtained using different combinations of data sets. In Section 3.1, we study the impact of a varying helium fraction and of the BBN consistency relation on the effective number of relativistic degrees of freedom. Section 3.2 analyzes the dependence of the neutrino abundances and masses on the varying lensing amplitude and on the matter power spectrum information. Finally in Section 3.3, we provide constraints on the neutrino perturbation parameters.

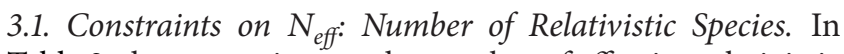
Table 2, the constraints on the number of effective relativistic degrees of freedom are shown with different priors.

First of all, in order to recall the effects of the number of effective relativistic degrees of freedom on CMB, we show in Figure 3 the degeneracies among $N_{\text {eff }}$ and the parameters that are directly measured by the $\mathrm{CMB}$ temperature power spectrum: the redshift of the equivalence $z_{\text {eq }}$, the angular scale of the sound horizon $\theta_{s}$, and the damping scale $\theta_{d}$. We can notice that $z_{\text {eq }}$ is proportional to the increase of $N_{\text {eff }}$ as expected from (3), while $\theta_{d}$ is correlated to $N_{\text {eff }}$ through the expansion rate at recombination $H$, because it scales as $\sqrt{H}$.

The inclusion of the $H_{0}$ prior moves the mean value of $N_{\text {eff }}$ towards a higher value and reduces the error on $N_{\text {eff }}$ $\left(N_{\text {eff }}=3.81 \pm 0.29\right.$ with respect to $N_{\text {eff }}=3.63 \pm 0.41,68 \%$ c.l.). The effect can be noticed in Figure 4 . The final result is a $\sim 2.6 \sigma$ evidence for an extra dark radiation component. Instead, applying the BBN consistency relation leads to a constraint on $N_{\text {eff }}$ much closer to the standard value than in the case of $Y_{\text {he }}$ fixed to 0.24 ; that is, $N_{\text {eff }}=3.44 \pm 0.35$ (68\% c.l.).

Finally, if we consider the helium fraction as a free parameter (last column of Table 2), the evidence for an extra number of relativistic degrees of freedom disappears and we obtain a milder constraint on $N_{\text {eff }}\left(N_{\text {eff }}=3.32 \pm 0.70,68 \%\right.$ c.l.) that makes it perfectly consistent with the prediction 
TABLE 2: Marginalized 68\% c.l. constraints on $N_{\text {eff }}$ in a standard cosmology with $N_{\text {eff }}$ massless neutrinos. In the second and in the fourth columns, we also apply a prior on the Hubble constant from the Hubble Space Telescope measurements. In the third and in the fourth columns, we apply the BBN consistency relation. In the last column, we also vary the helium fraction $Y_{\text {he }}$ and we show its marginalized $68 \%$ c.l.

\begin{tabular}{lccccc}
\hline Planck + WP + & Planck + WP \\
highL & highL $+H_{0}$ & $\begin{array}{c}\text { Planck + WP }+ \\
\text { highL }+ \text { BBNc }\end{array}$ & $\begin{array}{c}\text { Planck + WP + } \\
\text { highL }+H_{0}+ \\
\text { BBNc }\end{array}$ & $\begin{array}{c}\text { Planck + WP + } \\
\text { highL }\end{array}$ \\
\hline$N_{\text {eff }}$ & $3.63 \pm 0.41$ & $3.81 \pm 0.29$ & $3.44 \pm 0.35$ & $3.65 \pm 0.26$ & $3.32 \pm 0.70$ \\
\hline$Y_{\text {he }}$ & 0.24 & 0.24 & BBN & BBN & $0.260 \pm 0.036$ \\
\hline
\end{tabular}
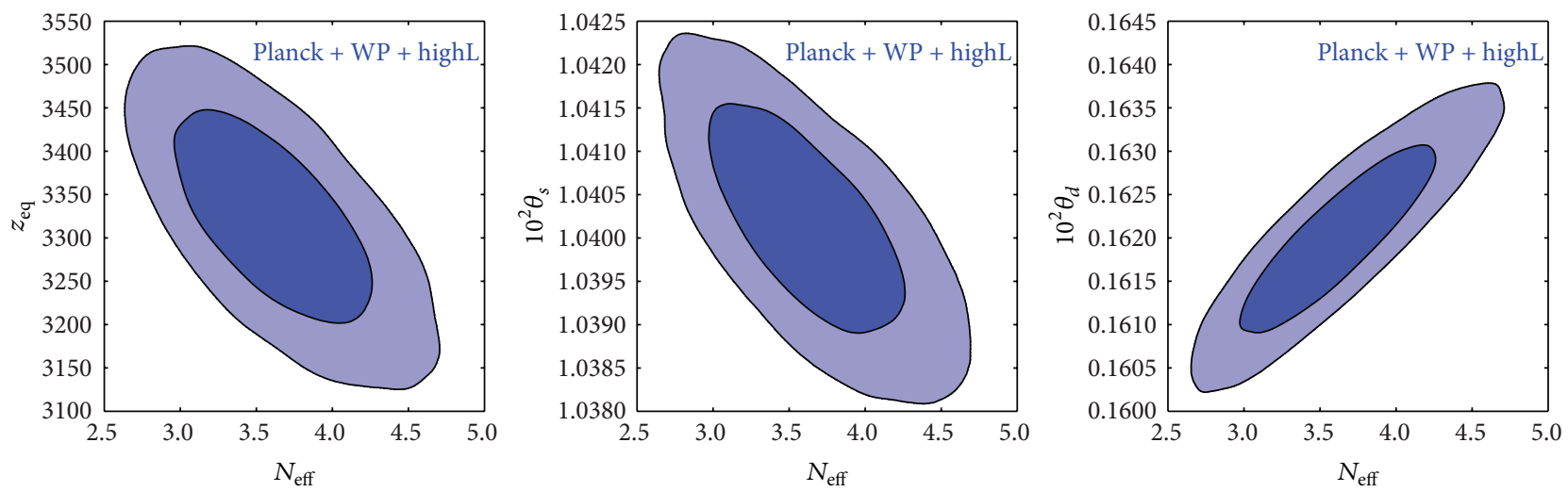

FIGURE 3: $68 \%$ and $95 \%$ c.l. 2D marginalized posterior in the plane $N_{\text {eff }}-H_{0}$.

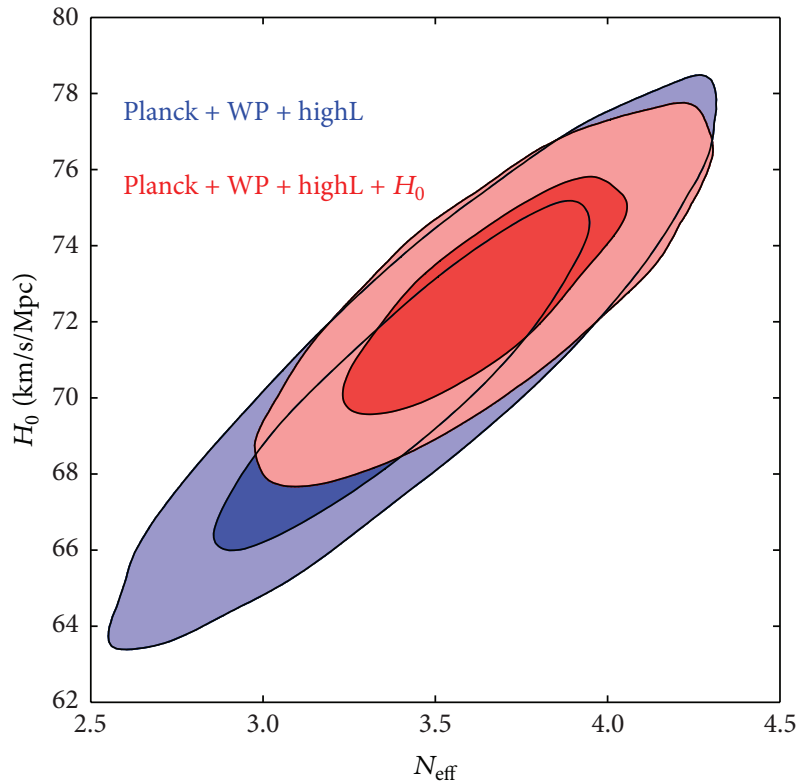

FIGURE 4: $68 \%$ and $95 \%$ c.l. 2D marginalized posterior in the plane $N_{\text {eff }}-H_{0}$.

of the Standard Model. Figure 5 shows the anticorrelation between $N_{\text {eff }}$ and $Y_{\text {he }}$ from CMB data (blue contours) and the $\mathrm{BBN}$ consistency relation among these two parameters (dotted line). We can notice that an increase in $N_{\text {eff }}$ requires a lower value of $Y_{\text {he }}$ to reproduce the same CMB power spectrum, as we have explained in Section 1.1. Concerning the comparison between the models with and without varying

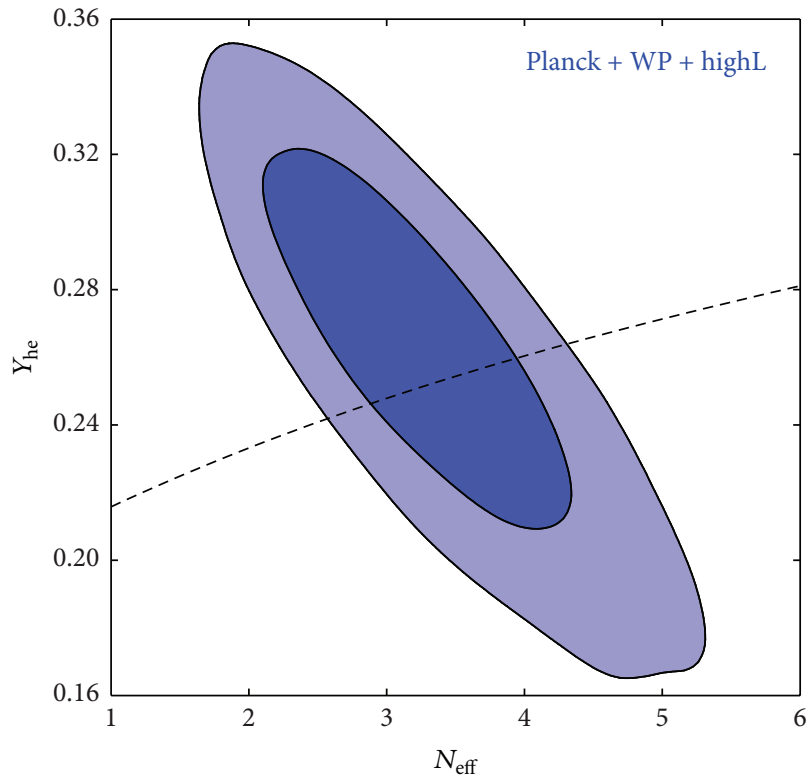

Figure 5: $68 \%$ and $95 \%$ c.l. 2D marginalized posterior in the plane $N_{\text {eff }}-Y_{\text {he }}$. Dotted line shows the BBN consistency relation.

the primordial helium fraction, the $\Delta \chi^{2}$ at the best fit point is negligible, meaning that a higher value of $Y_{\text {he }}$ is preferred by the data but a lower value can be accommodated by tuning the other parameters.

All the cases described above are illustrated in Figure 6 where the one-dimensional posterior of $N_{\text {eff }}$ is shown for the different cases of Table 2 . We can notice that both the 
TABLE 3: Marginalized 68\% c.l. constraints on $N_{\text {eff }}$ and $A_{L}$ and $95 \% \mathrm{cl}$ upper bounds on $\sum m_{v}$ in extended models with $N_{\text {eff }}$ massive neutrinos. We also include the lensing amplitude as a free parameter.

\begin{tabular}{|c|c|c|c|c|c|}
\hline & $\begin{array}{c}\text { Planck + WP + } \\
\text { highL }\end{array}$ & $\begin{array}{c}\text { Planck + WP + } \\
\text { highL }\end{array}$ & $\begin{array}{c}\text { Planck + WP + } \\
\text { highL }+H_{0}\end{array}$ & $\begin{array}{c}\text { Planck + WP + } \\
\text { highL + DR9 }\end{array}$ & $\begin{array}{c}\text { Planck + WP + } \\
\text { highL + DR9 + } \\
H_{0}\end{array}$ \\
\hline$N_{\text {eff }}$ & $3.38 \pm 0.36$ & $3.65 \pm 0.38$ & $3.81 \pm 0.28$ & $3.33 \pm 0.31$ & $3.65 \pm 0.26$ \\
\hline$\sum m_{v}[\mathrm{eV}]$ & $<0.64$ & $<1.03$ & $<0.66$ & $<0.66$ & $<0.51$ \\
\hline$A_{L}$ & 1 & $1.36 \pm 0.14$ & $1.36 \pm 0.14$ & $1.10 \pm 0.08$ & $1.10 \pm 0.07$ \\
\hline
\end{tabular}
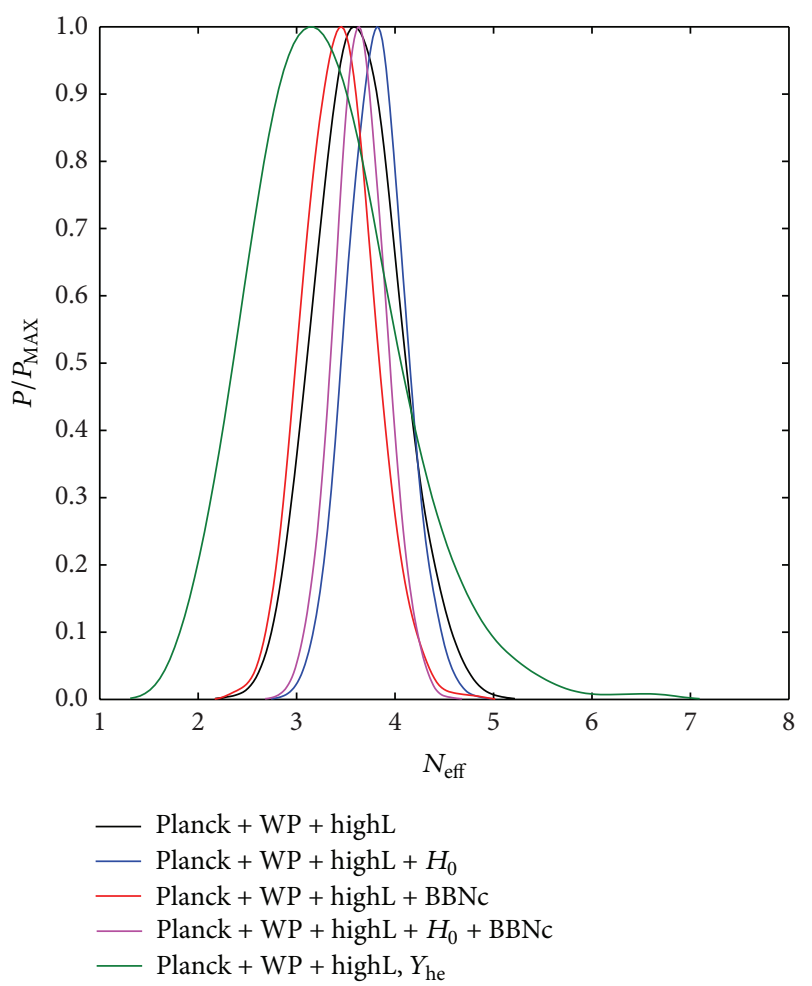

FIGURE 6: $1 \mathrm{D}$ posterior of $N_{\text {eff }}$. The different cases reported in Table 2 are shown: black line corresponds to Planck $+\mathrm{WP}+$ highL, blue line to Planck $+\mathrm{WP}+$ highL $+H_{0}$, red line to Planck $+\mathrm{WP}+$ highL + BBNc, and magenta line to Planck $+\mathrm{WP}+$ highL $+\mathrm{BBNc}$ $+H_{0}$. Finally, the green line refers to the analysis that includes also a varying $Y_{\text {he }}$.

inclusion of $H_{0}$ and BBN consistency narrow the posterior and reduce the error on $N_{\text {eff }}$. However, $H_{0}$ moves the best fit of $N_{\text {eff }}$ toward a higher value of the number of effective relativistic degrees of freedom, while BBN consistency prefers a lower value and brings back $N_{\text {eff }}$ closer to the standard value. In subsequent analyses, we will follow a conservative approach, applying the BBN consistency relation in all our MCMC analyses, accordingly also with Planck team strategy.

3.2. Constraints on $N_{\text {eff }}$ and $\sum m_{v}$ : Massive Neutrinos. The constraints on massive neutrinos are summarized in Table 3.

We also marginalize over the lensing amplitude and we study this effect in Figure 7 and in Figure 8 for our basic data set (Planck $+\mathrm{WP}+$ highL). As we already discussed in Section 2.2, Planck analysis points towards a value of the lensing amplitude higher than the standard one. This anomaly is confirmed by our results $A_{L}=1.36 \pm 0.14(68 \%$ c.l.) related to the model with a varying number of massive neutrinos. Nevertheless, including BOSS DR9, data shift the $A_{L}$ parameter towards a value consistent with the standard $A_{L}=1$ value within $2 \sigma\left(A_{L}=1.10 \pm 0.08,68 \%\right.$ c.l. $)$. It is clear from Figure $7(\mathrm{a})$ that the neutrino mass has a strong degeneracy with the lensing amplitude: allowing for a higher value of $A_{L}$ leading to a larger value of the neutrino mass; the $95 \%$ upper bound moves from $0.64 \mathrm{eV}$ to $1.03 \mathrm{eV}$. Figure 7(b) shows that there is no preferred direction for a correlation between $N_{\text {eff }}$ and $A_{L}$, but the side effect of the degeneracy among $\sum m_{v}$ and $A_{L}$ is also an increasing value of $N_{\text {eff }}(3.65 \pm$ 0.38 against $3.38 \pm 0.36,68 \%$ c.l.) related to the correlation among $N_{\text {eff }}$ and $\sum m_{v}$. This conclusion arises from Figure 8(a) where the increasing value of $A_{L}$ is located along the bisecting line in the plane $N_{\text {eff }}-\sum m_{v}$. We summarize the effect of the lensing amplitude on the neutrino parameters in Figure 8(b): a varying $A_{L}$ parameter will lead to a larger neutrino mass and, consequently, to a larger $N_{\text {eff }}$. Finally, we shall comment that a larger value of $A_{L}$ will provide a better fit to the data, lowering the $\chi^{2}$ by 4.2 units.

Concerning the effects of external non-CMB data sets, we include in the analyses of a $\Lambda$ CDM model with massive neutrinos and a varying lensing amplitude the $H_{0}$ prior and the BOSS DR9 data. On one hand with the inclusion of the $H_{0}$ prior, we obtain a better constraint on $N_{\text {eff }}$, driving $N_{\text {eff }}$ from $N_{\text {eff }}=3.65 \pm 0.38$ to $N_{\text {eff }}=3.81 \pm 0.28$ (68\% c.l.). So the combination of the data sets Planck $+\mathrm{WP}+$ highL $+\mathrm{H}_{0}$ provides a stronger evidence $(2.7 \sigma)$ for an extra dark radiation component. On the other hand, $H_{0}$ leads to tighter constraints on the $95 \%$ c.l. upper bound of the sum of neutrino masses, moving it from $\sum m_{v}<1.03 \mathrm{eV}$ to $\sum m_{v}<$ $0.66 \mathrm{eV}$ at $95 \%$ c.l. (see Figure 9(a)). The same effect on $\sum m_{v}$ can be achieved by including BOSS DR9, but in this case $N_{\text {eff }}$ remains close to the standard value $N_{\text {eff }}=3.26 \pm 0.30$ (68\% c.l.) (see Figure 9(b)). The joint effect of adding both an $H_{0}$ prior and the galaxy clustering information from BOSS DR9 is shown in Figure 9(c): the 95\% upper bound on the sum of neutrino masses is tightened both by the prior on $\mathrm{H}_{0}$ and the BOSS DR9 galaxy clustering information, and an extra dark radiation component is favored at $2.3 \sigma$ level $\left(N_{\text {eff }}=3.65 \pm 0.26,68 \%\right.$ c.l. $)$.

\subsection{Constraints on $c_{e f f}^{2}$ and $c_{v i s}^{2}$ : Perturbation Parameters.}

Table 4 reports the constraints on the perturbation parameters of a varying number of relativistic species. The neutrino 


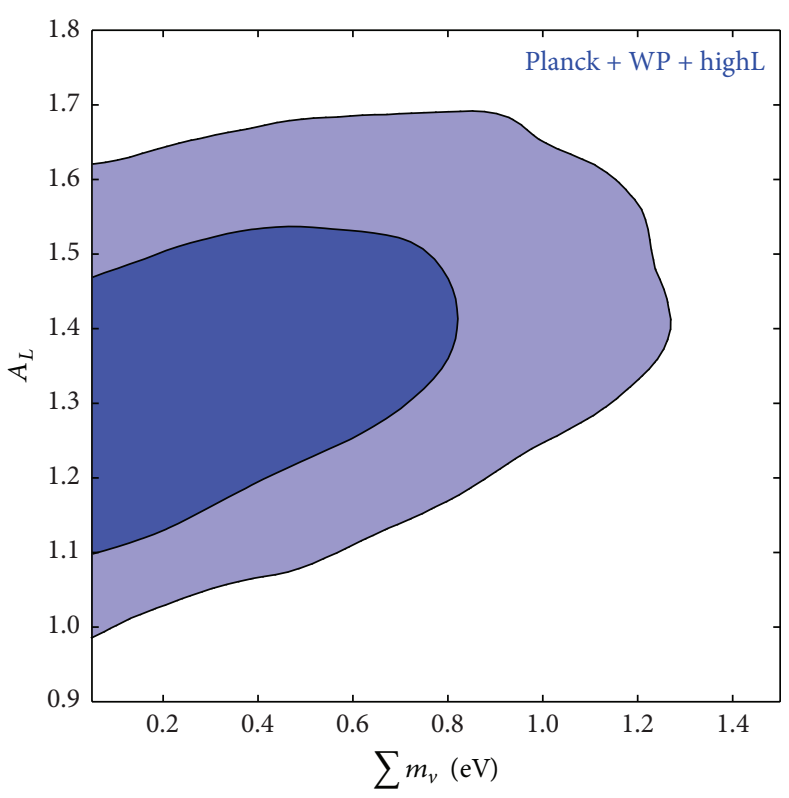

(a)

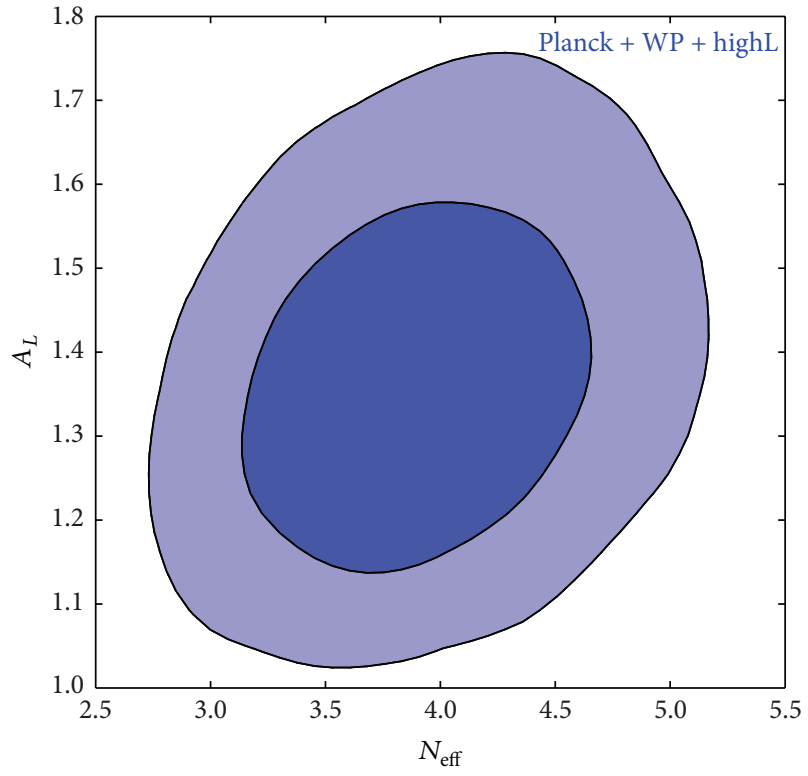

(b)

Figure 7: $68 \%$ and 95\% c.l. 2D marginalized posterior in the plane $\sum m_{v}-A_{L}$ (a) and in the plane $N_{\text {eff }}-A_{L}$ (b).

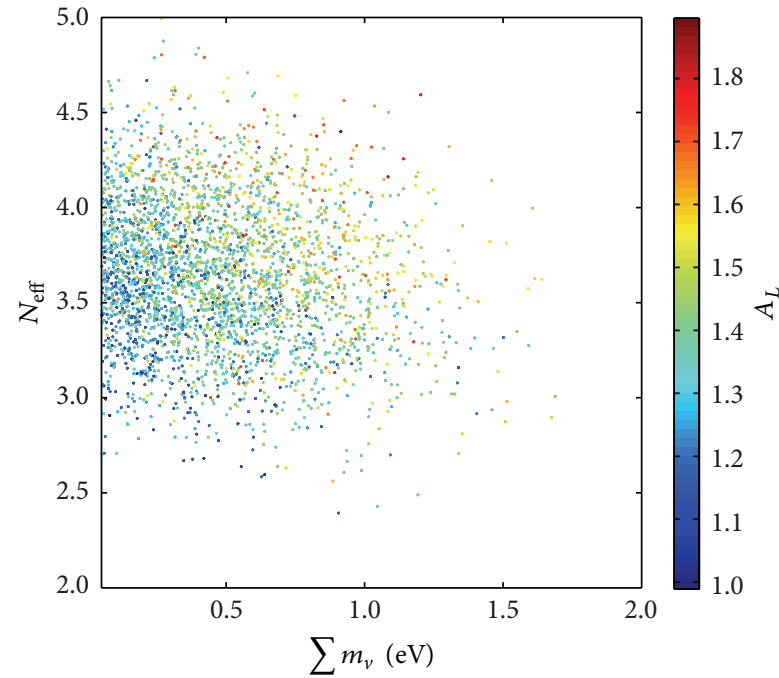

(a)

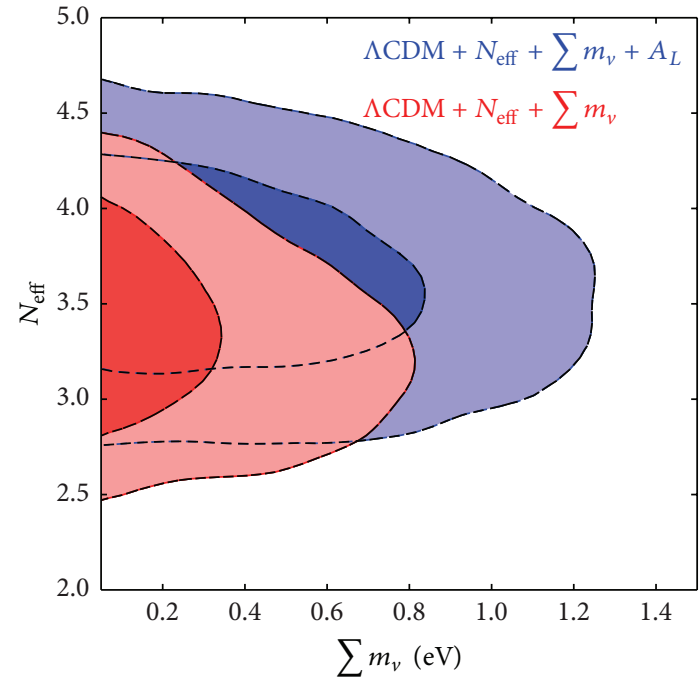

(b)

FIGURE 8: (a) Scatter plot in the $\sum m_{v}-N_{\text {eff }}$ plane with points colored by the value of the $A_{L}$ parameter (second column of Table 3). (b) 68\% and $95 \%$ c.l. $2 \mathrm{D}$ marginalized posterior in the plane $\sum m_{v}-N_{\text {eff }}$; blue contours refer to the case with a varying lensing amplitude (second column of Table 3 ) and red contours illustrate the $A_{L}=1$ case (first column of Table 3 ).

perturbation parameters are not strongly affected by the inclusion of the $H_{0}$ prior: the constraints on $c_{\text {eff }}^{2}$ and $c_{\mathrm{vis}}^{2}$ remain almost the same. Interestingly both the effective sound speed and the viscosity parameter show a deviation from the standard value 0.33 having $c_{\text {eff }}^{2}=0.309 \pm 0.012$ and $c_{\mathrm{vis}}^{2}=0.56 \pm 0.17$ at $68 \%$ c.l. for the basic data set Planck + WP + highL, consistent with the results of [84].
Furthermore, we can notice that varying the neutrino perturbation parameters does not change our conclusions on the effective number of relativistic species; the bounds on $N_{\text {eff }}$ turn out to be almost the same as those reported in Table 2: varying $c_{\text {eff }}^{2}$ and $c_{\text {vis }}^{2}$, we get $N_{\text {eff }}=3.40 \pm 0.34$ ( $68 \%$ c.l.), while we obtained $N_{\text {eff }}=3.44 \pm 0.35$ (68\% c.l.) with standard $c_{\text {eff }}^{2}$ and $c_{\mathrm{vis}}^{2}$. 


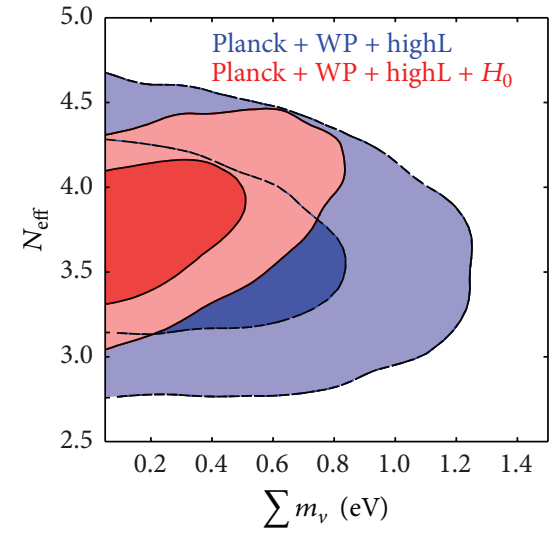

(a)

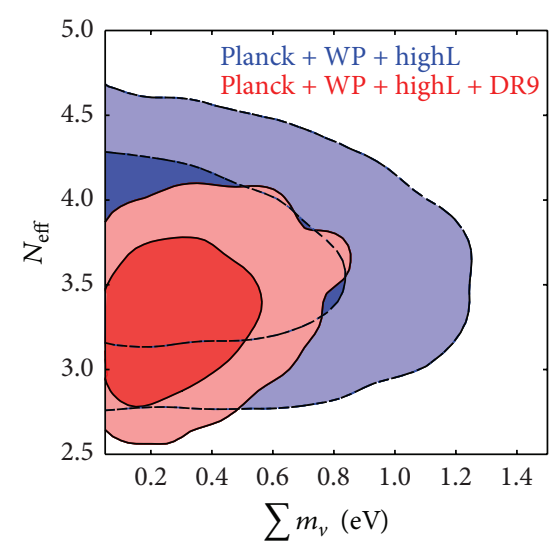

(b)

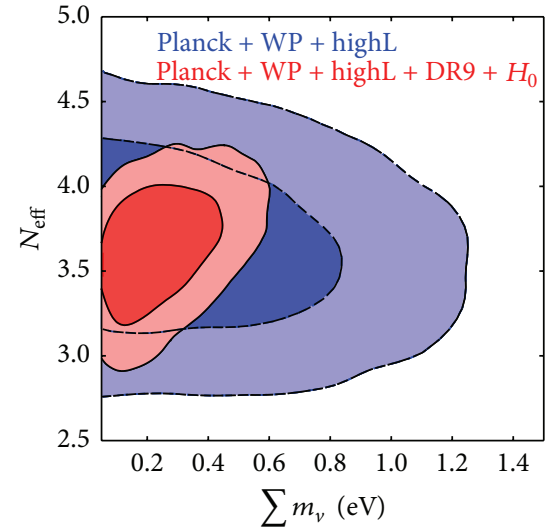

(c)

FIGURE 9: $68 \%$ and 95\% c.l. 2D marginalized posterior in the $\sum m_{\nu}-N_{\text {eff }}$ plane. Blue contours refer to the constraints from the combination of Planck + WP + highL and red contours include also $H_{0}$ (a), BOSS DR9 (b), and $H_{0}$ and BOSS DR9 (c).

\section{Future Constraints}

We present here a forecast of the impact of the Euclid survey [85] in constraining $N_{\text {eff }}$ and $\sum m_{v}$ (for a recent and complete analysis see [70]). We perform a Fisher matrix analysis following the prescription of [86]. The fiducial values of the standard cosmological parameters are fixed at the best fit values obtained by Planck [24] (see Table 5). Concerning the neutrino parameters, the fiducial value of $N_{\text {eff }}$ is fixed at the standard cosmological value $N_{\text {eff }}=3.046$, while the neutrino mass fiducial value is $\sum m_{v}=0.2 \mathrm{eV}$ (we recall here that the minimum mass sum in the inverted hierarchy is $0.11 \mathrm{eV}$, while in the normal hierarchy it is $0.06 \mathrm{eV}$ ). Furthermore, we add priors on the standard cosmological parameters from Planck results [24].

The $1 \sigma$ marginalized errors on the parameters of the two different fiducial cosmological models (with either massless or massive neutrinos) are reported in Table 6 . The relative errors show that Euclid will improve the constraints on both $N_{\text {eff }}$ and $\sum m_{v}$. Nevertheless, even Euclid will not be able to reveal the hierarchy, it would provide a detection of the neutrino mass sum only if $\sum m_{v}>0.1 \mathrm{eV}$ [87].

\section{Conclusions}

The newly released Planck data have provided us with an extremely precise picture of the cosmic microwave background, confirming the standard $\Lambda$ CDM model. However, the exact properties of the dark sector are still under discussion and, in particular, there is no strong argument against the existence of a dark radiation component. On the contrary, combining CMB data with measurements of galaxy clustering and of the Hubble constant leads to an evidence for a nonstandard number of relativistic species.

In this paper we have illustrated the effects of an additional relativistic component on the temperature power spectrum and we have reviewed the most promising models to explain the presence of this component: sterile neutrinos,
TABLE 4: Marginalized $68 \%$ constraints on $N_{\text {eff }}, c_{\text {eff }}^{2}$, and $c_{\mathrm{vis}}^{2}$.

\begin{tabular}{lcc}
\hline & $\begin{array}{c}\text { Planck + WP + } \\
\text { highL }\end{array}$ & $\begin{array}{c}\text { Planck }+ \text { WP + } \\
\text { highL }+H_{0}\end{array}$ \\
\hline$N_{\text {eff }}$ & $3.40 \pm 0.34$ & $3.56 \pm 0.26$ \\
\hline$c_{\text {eff }}^{2}$ & $0.309 \pm 0.012$ & $0.310 \pm 0.012$ \\
$c_{\text {vis }}^{2}$ & $0.56 \pm 0.17$ & $0.57 \pm 0.17$ \\
\hline
\end{tabular}

TABLE 5: Fiducial values of the cosmological parameters considered in the forecast analysis.

\begin{tabular}{lc}
\hline Parameter & Fiducial value \\
\hline$\omega_{b}$ & 0.02207 \\
$\omega_{\text {cdm }}$ & 0.1203 \\
$h$ & 0.671 \\
$\ln \left(10^{10} A_{s}\right)$ & 3.098 \\
$n_{s}$ & 0.962 \\
\hline$N_{\text {eff }}[\mathrm{eV}]$ & 3.046 \\
$\sum m_{v}$ & 0.2 \\
\hline
\end{tabular}

axions, decay of massive particles, and interactions between dark matter and dark radiation sectors.

We have focused on the hypothesis of a link between cosmology and neutrino physics that can explain the cosmologically inferred excess in the number of relativistic species in terms of sterile neutrinos whose existence could explain some short baseline neutrino oscillations results. In this framework, we have updated the cosmological constraints on massive neutrinos including the new Planck CMB data and the matter power spectrum from BOSS DR9. Including also a prior on the Hubble constant from the Hubble Space Telescope measurements, our results show a preference for a nonstandard number of neutrino species at $2.3 \sigma$ with $N_{\text {eff }}=$ $3.65 \pm 0.26$ at $68 \%$ c.l. and an upper bound on the sum of neutrino masses of $0.51 \mathrm{eV}$ at $95 \%$ c.l.

However, the relevance of these cosmological constraints on dark radiation depends on the model and on the data sets. 
TABLE 6: $1 \sigma$ marginalized errors and relative errors for all parameters considering the two different fiducial cosmological models described in the text: $\Lambda \mathrm{CDM}$ model with either massless or massive neutrinos.

\begin{tabular}{|c|c|c|c|c|}
\hline & \multicolumn{4}{|c|}{ Euclid + Planck prior } \\
\hline & \multicolumn{2}{|c|}{$\Lambda \mathrm{CDM}+N_{\mathrm{eff}}$} & \multicolumn{2}{|c|}{$\Lambda \mathrm{CDM}+N_{\mathrm{eff}}+\sum m_{v}$} \\
\hline & $1 \sigma$ & rel. err. & $1 \sigma$ & rel. err. \\
\hline$\omega_{b}$ & 0.00031 & $1.4 \%$ & 0.00031 & $1.4 \%$ \\
\hline$\omega_{\mathrm{cdm}}$ & 0.0021 & $1.7 \%$ & 0.0022 & $1.8 \%$ \\
\hline$h$ & 0.007 & $1.0 \%$ & 0.007 & $1.1 \%$ \\
\hline $\ln \left(10^{10} A_{s}\right)$ & 0.064 & $2.1 \%$ & 0.072 & $2.3 \%$ \\
\hline$n_{s}$ & 0.009 & $0.9 \%$ & 0.009 & $0.9 \%$ \\
\hline$N_{\text {eff }}$ & 0.23 & $7.5 \%$ & 0.29 & $9.7 \%$ \\
\hline$\sum m_{v}[\mathrm{eV}]$ & - & - & 0.10 & $51.4 \%$ \\
\hline
\end{tabular}

We have stressed the impact of the lensing amplitude on these results; the inclusion of a varying lensing amplitude drives the results towards a more statistically significant detection of dark radiation.

Concerning the data sets, the $H_{0}$ prior also leads to a better constraint on $N_{\text {eff }}$. The former effect is related to the $2.5 \sigma$ tension among Planck and HST measurements of the Hubble constant that must be fixed [88].

Finally our results confirm a significant deviation from the standard values $\left(c_{\text {eff }}^{2}=1 / 3\right.$ and $\left.c_{\text {vis }}^{2}=1 / 3\right)$ expected for a free streaming dark radiation component. We find $c_{\text {eff }}^{2}=$ $0.309 \pm 0.012$ and $c_{\text {vis }}^{2}=0.56 \pm 0.17$ at $68 \%$ c.l., allowing for further consideration on the nature of dark radiation.

In conclusion, there is still ample room for interesting new discoveries of physics beyond the standard model in the form of dark radiation.

\section{Acknowledgment}

The authors acknowledge the European ITN project Invisibles (FP7-PEOPLE-2011-ITN, PITN-GA-2011-289442INVISIBLES).

\section{References}

[1] D. V. Forero, M. Tortola, and J. W. F. Valle, "Global status of neutrino oscillation parameters after Neutrino-2012," Physical Review D, vol. 86, Article ID 073012, 2012.

[2] G. L. Fogli, E. Lisi, A. Marrone, D. Montanino, A. Palazzo, and A. M. Rotunno, "Global analysis of neutrino masses, mixings, and phases: entering the era of leptonic CP violation searches," Physical Review D, vol. 86, Article ID 013012, 10 pages, 2012.

[3] S. Hannestad, "Neutrino physics from precision cosmology," Progress in Particle and Nuclear Physics, vol. 65, no. 2, pp. 185208, 2010.

[4] Y. Y. Y. Wong, "Neutrino mass in cosmology: status and prospects," Annual Review of Nuclear and Particle Science, vol. 61, pp. 69-98, 2011.

[5] A. Osipowicz, H. Blumer, G. Drexlin et al., "KATRIN: a next generation tritium beta decay experiment with sub-eV sensitivity for the electron neutrino mass," http://arxiv.org/abs/hepex/0109033.
[6] S. Riemer-Sørensen, D. Parkinson, and T. M. Davis, "Combining Planck with large scale structure gives strong neutrino mass constraint," http://arxiv.org/abs/1306.4153.

[7] E. Giusarma, R. de Putter, S. Ho, and O. Mena, "Constraints on neutrino masses from Planck and Galaxy Clustering data," Physical Review D, vol. 88, Article ID 063515, 2013.

[8] J. Lesgourgues and S. Pastor, "Neutrino mass from cosmology," Advances in High Energy Physics, vol. 2012, Article ID 608515, 34 pages, 2012.

[9] M. C. Gonzalez-Garcia, M. Maltoni, and J. Salvado, "Direct determination of the solar neutrino fluxes from solar neutrino data," Journal of High Energy Physics, vol. 2010, article 072, 2010.

[10] A. Smith, M. Archidiacono, A. Cooray, F. De Bernardis, A. Melchiorri, and J. Smidt, "Impact of assuming flatness in the determination of neutrino properties from cosmological data," Physical Review D, vol. 85, Article ID 123521, 6 pages, 2012.

[11] S. Hannestad, "Neutrino masses and the dark energy equation of state:relaxing the cosmological neutrino mass bound," Physical Review Letters, vol. 95, Article ID 221301, 2005.

[12] G. Abbiendi, C. Ainsley, P. F. Akesson et al., "Precise determination of the $\mathrm{Z}$ resonance parameters at LEP: 'Zedometry", European Physical Journal C, vol. 19, no. 4, pp. 587-651, 2001.

[13] G. Mangano, G. Miele, S. Pastor, T. Pinto, O. Pisanti, and P. D. Serpico, "Relic neutrino decoupling including flavour oscillations," Nuclear Physics B, vol. 729, no. 1-2, pp. 221-234, 2005.

[14] J. L. Sievers, R. A. Hlozek, M. R. Nolta et al., "The atacama cosmology telescope: cosmological parameters from three seasons of data," Journal of Cosmology and Astroparticle Physics, vol. 1310, Article ID 060, 2013.

[15] Z. Hou, C. L. Reichardt, K. T. Story et al., "Constraints on cosmology from the cosmic microwave background power spectrum of the 2500-square degree SPT-SZ survey," http://arxiv.org/abs/1212.6267.

[16] M. Archidiacono, E. Giusarma, A. Melchiorri, and O. Mena, "Neutrino and dark radiation properties in light of recent CMB observations," Physical Review D, vol. 87, Article ID 103519, 10 pages.

[17] S. Riemer-Sørensen, D. Parkinson, and T. M. Davis, "What is half a neutrino? Reviewing cosmological constraints on neutrinos and dark radiation," http://arxiv.org/abs/1301.7102.

[18] D. Schlegel, M. White, D. Eisenstein et al., "The Baryon oscillation spectroscopic survey: precision measurements of the absolute cosmic scale," http://arxiv.org/abs/0902.4680. 
[19] K. S. Dawson, D. J. Schlegel, C. P. Ahn et al., "The Baryon oscillation spectroscopic survey of SDSS-III," http://arxiv.org/ abs/1208.0022.

[20] N. Padmanabhan, X. Xu, D. J. Eisenstein et al., "A 2 \% distance to $\mathrm{z}=0.35$ by reconstructing Baryon Acoustic oscillationsI :methods and application to the sloan digital sky survey," Monthly Notices of the Royal Astronomical Society, vol. 427, no. 3, article 2132, 2012.

[21] F. Beutler, C. Blake, M. Colless et al., "The 6dF galaxy survey: baryon acoustic oscillations and the local hubble constant," Monthly Notices of the Royal Astronomical Society, vol. 416, pp. 3017-3032, 2011.

[22] C. Blake, E. Kazin, F. Beutler et al., "The WiggleZ dark energy survey: mapping the distance-redshift relation with baryon acoustic oscillations," Monthly Notices of the Royal Astronomical Society, vol. 418, pp. 1707-1724, 2011.

[23] A. G. Riess, L. Macri, S. Casertano et al., "A 3\% solution: determination of the hubble constant with the hubble space telescope and wide field camera distance 3," The Astrophysical Journal, vol. 730, p. 119, 2011.

[24] P. A. R. Ade, N. Aghanim, C. Armitage-Caplan et al., "Planck 2013 results. XVI. Cosmological parameters," http://arxiv.org/ abs/1303.5076.

[25] Z. Hou, R. Keisler, L. Knox, M. Millea, and C. Reichardt, "How massless neutrinos affect the cosmic microwave background damping tail," http://arxiv.org/abs/1104.2333 .

[26] M. Zaldarriaga and D. D. Harari, "Analytic approach to the polarization of the cosmic microwave background in flat and open universes," Physical Review D, vol. 52, no. 6, pp. 3276-3287, 1995.

[27] R. Bowen, S. H. Hansen, A. Melchiorri, J. Silk, and R. Trotta, "The impact of an extra background of relativistic particles on the cosmological parameters derived from the cosmic microwave background," Monthly Notices of the Royal Astronomical Society, vol. 334, no. 4, pp. 760-768, 2002.

[28] S. Bashinsky and U. Seljak, "Signatures of relativistic neutrinos in CMB anisotropy and matter clustering," Physical Review D, vol. 69, no. 8, Article ID 083002, 2004.

[29] S. Hannestad, "Structure formation with strongly interacting neutrinos-implications for the cosmological neutrino mass bound," Journal of Cosmology and Astroparticle Physics, vol. 0502, article 011, 2005.

[30] W. Hu and N. Sugiyama, "Small-scale cosmological perturbations: an analytic approach," The Astrophysical Journal Letters, vol. 471, no. 2, pp. 542-570, 1996.

[31] A. Aguilar-Arevalo, L. B. Auerbach, R. L. Burman et al., "Evidence for neutrino oscillations from the observation of $\bar{v}_{e}$ appearance in a $\bar{v}_{\mu}$ beam," Physical Review D, vol. 64, Article ID 112007, 22 pages, 2001.

[32] S. M. Bilenky, C. Giunti, and W. Grimus, "Neutrino mass spectrum from the results of neutrino oscillation experiments," European Physical Journal C, vol. 1, pp. 247-253, 1998.

[33] V. D. Barger, T. J. Weiler, and K. Whisnant, "Four-way neutrino oscillations," Physics Letters B, vol. 427, pp. 97-104, 1998.

[34] M. Sorel, J. M. Conrad, and M. H. Shaevitz, "Combined analysis of short-baseline neutrino experiments in the $(3+1)$ and $(3+2)$ sterile neutrino oscillation hypotheses," Physical Review D, vol. 70, no. 7, Article ID 073004, 2004.

[35] S. Goswami and W. Rodejohann, "MiniBooNE results and neutrino schemes with 2 sterile neutrinos: possible mass orderings and observables related to neutrino masses," Journal of High Energy Physics, vol. 0710, article 073, 2007.
[36] A. A. Aguilar-Arevalo, B. C. Brown, L. Bugel et al., "Improved Search for $\bar{v}_{\mu} \rightarrow \bar{v}_{e}$ Oscillations in the MiniBooNE Experiment," Physical Review Letters, vol. 110, Article ID 161801, 6 pages, 2013.

[37] J. Kopp, P. A. N. Machado, M. Maltoni, and T. Schwetz, "Sterile neutrino oscillations: the global picture," Journal of High Energy Physics, vol. 1305, article 050, 2013.

[38] J. M. Conrad, C. M. Ignarra, G. Karagiorgi, M. H. Shaevitz, and J. Spitz, "Sterile neutrino fits to short-baseline neutrino oscillation measurements," Advances in High Energy Physics, vol. 2013, Article ID 163897, 26 pages, 2013.

[39] S. Dodelson, A. Melchiorri, and A. Slosar, "Is cosmology compatible with sterile neutrinos?" Physical Review Letters, vol. 97, no. 4, Article ID 041301, 2006.

[40] A. Melchiorri, O. Mena, S. Palomares-Ruiz, S. Pascoli, A. Slosar, and M. Sorel, "Sterile neutrinos in light of recent cosmological and oscillation data: a multi-flavor scheme approach," Journal of Cosmology and Astroparticle Physics, vol. 0901, article 036, 2009.

[41] S. Hannestad, I. Tamborra, and T. Tram, "Thermalisation of light sterile neutrinos in the early universe," Journal of Cosmology and Astroparticle Physics, vol. 1207, article 025, 2012.

[42] M. Archidiacono, N. Fornengo, C. Giunti, S. Hannestad, and A. Melchiorri, "Sterile neutrinos: cosmology vs short-baseline experiments," http://arxiv.org/abs/1302.6720.

[43] A. Mirizzi, G. Mangano, N. Saviano et al., "The strongest bounds on active-sterile neutrino mixing after Planck data," Physics Letters B, vol. 726, pp. 8-14, 2013.

[44] D. Hooper, F. S. Queiroz, and N. Y. Gnedin, "Nonthermal dark matter mimicking an additional neutrino species in the early universe," Physical Review D, vol. 85, no. 6, Article ID 063513, 2012.

[45] C. Kelso, S. Profumo, and F. S. Queiroz, "Nonthermal WIMPs as "dark radiation" in light of ATACAMA, SPT, WMAP9, and Planck," Physical Review D, vol. 88, Article ID 023511, 8 pages, 2013.

[46] C. Brust, D. E. Kaplan, and M. T. Walters, "New light species and the CMB," http://arxiv.org/abs/1303.5379.

[47] J. E. Kim, "Weak-interaction singlet and strong CP invariance," Physical Review Letters, vol. 43, no. 2, pp. 103-107, 1979.

[48] M. A. Shifman, A. I. Vainshtein, and V. I. Zakharov, "Can confinement ensure natural CP invariance of strong interactions?" Nuclear Physics, Section B, vol. 166, no. 3, pp. 493-506, 1980.

[49] C. A. Baker, D. D. Doyle, P. Geltenbort et al., "Improved experimental limit on the electric dipole moment of the neutron," Physical Review Letters, vol. 97, Article ID 131801, 4 pages, 2006.

[50] R. D. Peccei and H. R. Quinn, "CP conservation in the presence of pseudoparticles," Physical Review Letters, vol. 38, no. 25, pp. 1440-1443, 1977.

[51] R. D. Peccei and H. R. Quinn, "Constraints imposed by CP conservation in the presence of pseudoparticles," Physical Review D, vol. 16, pp. 1791-1797, 1977.

[52] S. Hannestad, A. Mirizzi, and G. Raffelt, "A new cosmological mass limit on thermal relic axions," Journal of Cosmology and Astroparticle Physics, vol. 0507, article 002, 2005.

[53] S. Hannestad, A. Mirizzi, G. G. Raelt, and Y. Y. Y. Wong, "Cosmological constraints on neutrino plus axion hot dark matter," Journal of Cosmology and Astroparticle Physics, vol. 0708, article 015, 2007.

[54] A. Melchiorri, O. Mena, and A. Slosar, "Improved cosmological bound on the thermal axion mass," Physical Review D, vol. 76, no. 4, Article ID 041303, 2007. 
[55] M. Archidiacono, S. Hannestad, A. Mirizzi, G. Raffelt, and Y. Y. Y. Wong, "Axion hot dark matter bounds after Planck," Journal of Cosmology and Astroparticle Physics, vol. 1310, Article ID 020, 2013.

[56] M. Blennow, B. Dasgupta, E. Fernandez-Martinez, and N. Rius, "Aidnogenesis via leptogenesis and dark sphalerons," Journal of High Energy Physics, vol. 1103, article 014, 2011.

[57] M. Blennow, E. Fernandez-Martinez, O. Mena, J. Redondo, and P. Serra, "Asymmetric dark matter and dark radiation," Journal of Cosmology and Astroparticle Physics, vol. 1207, article 022, 2012.

[58] U. Franca, R. A. Lineros, J. Palacio, and S. Pastor, "Probing interactions within the dark matter sector via extra radiation contributions," Physical Review D, vol. 87, Article ID 123521, 6 pages.

[59] C. Boehm, M. J. Dolan, and C. McCabe, "Increasing $N_{\text {eff }}$ with particles in thermal equilibrium with neutrinos," Journal of Cosmology and Astroparticle Physics, vol. 1212, article 027, 2012.

[60] Cl. Boehm, M. J. Dolan, and C. McCabe, "A lower bound on the mass of cold thermal dark matter from planck," Journal of Cosmology and Astroparticle Physics, vol. 1308, article 041, 2013.

[61] G. Hinshaw, D. Larson, E. Komatsu et al. et al., "Nineyear Wilkinson Microwave Anisotropy Probe (WMAP) observations: cosmological parameter results," http://arxiv.org/abs/ 1212.5226 .

[62] K. T. Story, C. L. Reichardt, Z. Hou et al., "A measurement of the cosmic microwave background damping tail from the 2500square-degree SPT-SZ survey," http://arxiv.org/abs/1210.7231.

[63] E. Di Valentino, A. Melchiorri, and O. Mena, "Dark Radiation candidates after Planck," http://arxiv.org/abs/1304.5981.

[64] G. Mangano, A. Melchiorri, P. Serra, A. Cooray, and M. Kamionkowski, "Cosmological bounds on dark-matterneutrino interactions," Physical Review D, vol. 74, no. 4, Article ID 043517, 2006.

[65] R. Diamanti, E. Giusarma, O. Mena, M. Archidiacono, and A. Melchiorri, "Dark radiation and interacting scenarios," Physical Review D, vol. 87, Article ID 063509, 8 pages, 2013.

[66] A. Lewis and S. Bridle, "Cosmological parameters from CMB and other data: a Monte Carlo approach," Physical Review D, vol. 66, Article ID 103511, 16 pages, 2002.

[67] A. Lewis, A. Challinor, and A. Lasenby, "Efficient computation of cosmic microwave background anisotropies in closed Friedmann-Robertson-Walker models," The Astrophysical Journal Letters, vol. 538, no. 2, pp. 473-476, 2000.

[68] C. P. Ahn, R. Alexandroff, C. Allende Prieto et al., "The ninth data release of the sloan digital sky survey: first spectroscopic data from the SDSS-III baryon oscillation spectroscopic survey," The Astrophysical Journal Supplement, vol. 203, p. 21, 2012.

[69] D. J. Eisenstein, D. H. Weinberg, E. Agolt et al., "SDSS-III: massive spectroscopic surveys of the distant universe, the milky way, and extra-solar planetary systems," The Astronomical Journal, vol. 142, article 72, 2011.

[70] T. Basse, O. E. Bjaelde, J. Hamann, S. Hannestad, and Y. Y. Y. Wong, "Dark energy and neutrino constraints from a future EUCLID-like survey," http://arxiv.org/abs/1304.2321.

[71] Y. I. Izotov and T. X. Thuan, "The primordial abundance of4He: evidence for non-standard big bang nucleosynthesis," The Astrophysical Journal Letters, vol. 710, no. 1, pp. L67-L71, 2010.

[72] G. Steigman, "Neutrinos and big bang nucleosynthesis," Advances in High Energy Physics, vol. 2012, Article ID 268321, 24 pages, 2012.
[73] W. Fischler and J. Meyers, "Dark radiation emerging after big bang nucleosynthesis?” Physical Review D, vol. 83, Article ID 063520, 5 pages, 2011.

[74] O. E. Bjaelde, S. Das, and A. Moss, "Origin of $\Delta N_{\text {eff }}$ as a result of an interaction between dark radiation and dark matter," Journal of Cosmology and Astroparticle Physics, vol. 1210, article 017, 2012.

[75] J. Hasenkamp, "Dark radiation from the axino solution of the gravitino problem," Physics Letters B, vol. 707, no. 1, pp. 121-128, 2012.

[76] L. M. Krauss, C. Lunardini, and C. Smith, "Neutrinos, WMAP, and BBN," http://arxiv.org/abs/1009.4666.

[77] J. Hamann, J. Lesgourgues, and G. Mangano, "Using big bang nucleosynthesis in cosmological parameter extraction from the cosmic microwave background: a forecast for PLANCK," Journal of Cosmology and Astroparticle Physics, vol. 0803, article 004, 2008.

[78] O. Pisanti, A. Cirillo, S. Esposito et al., "PArthENoPE: public algorithm evaluating the nucleosynthesis of primordial elements," Computer Physics Communications, vol. 178, no. 12, pp. 956-971, 2008.

[79] N. Said, E. Di Valentino, and M. Gerbino, "Planck constraints on the effective neutrino number and the $\mathrm{CMB}$ power spectrum lensing amplitude," vol. 88, Article ID 023513, 6 pages, 2013.

[80] H. U. Wayne, "Structure formation with generalized dark matter," The Astrophysical Journal Letters, vol. 506, no. 2, pp. 485-494, 1998.

[81] R. Trotta and A. Melchiorri, "Indication for primordial anisotropies in the neutrino background from the Wilkinson Microwave Anisotropy Probe and the sloan digital sky survey," Physical Review Letters, vol. 95, no. 1, Article ID 011305, 2005.

[82] M. Archidiacono, E. Calabrese, and A. Melchiorri, "Case for dark radiation," Physical Review D, vol. 84, no. 12, Article ID 123008, 2011.

[83] T. L. Smith, S. Das, and O. Zahn, "Constraints on neutrino and dark radiation interactions using cosmological observations," Physical Review D, vol. 85, Article ID 023001, 6 pages, 2012.

[84] M. Gerbino, E. Di Valentino, and N. Said, "Neutrino anisotropies after Planck," Physical Review D, vol. 88, Article ID 063538, 8 pages, 2013.

[85] A. Refregier, A. Amara, T. D. Kitching et al., "Euclid imaging consortium science book," http://arxiv.org/abs/arXiv:1001.0061.

[86] E. Giusarma, M. Corsi, M. Archidiacono et al., "Constraints on massive sterile neutrino species from current and future cosmological data," Physical Review D, vol. 83, no. 11, Article ID 115023, 2011.

[87] C. Carbone, "Neutrino mass from future large scale structure surveys," Nuclear Physics B, vol. 237-238, pp. 50-53, 2013.

[88] V. Marra, L. Amendola, I. Sawicki, and W. Valkenburg, "Cosmic variance and the measurement of the local Hubble parameter," Physical Review Letters, vol. 110, Article ID 241305, 5 pages, 2013. 

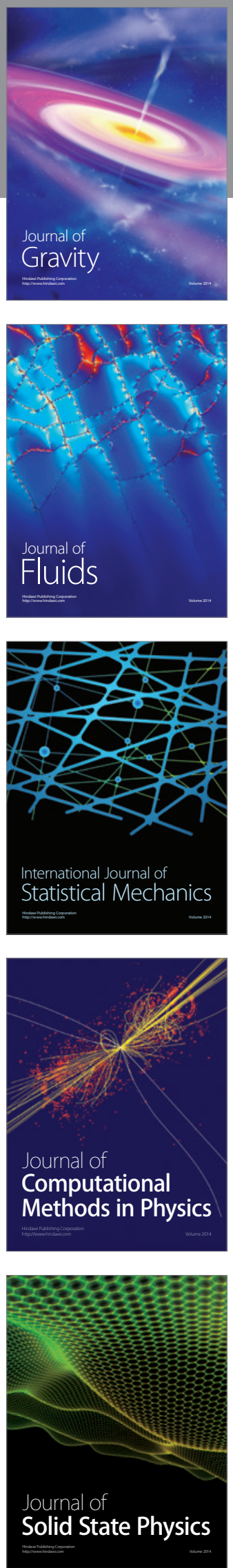

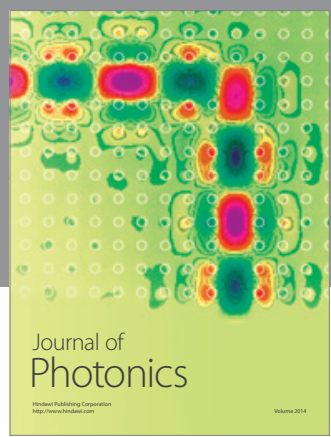

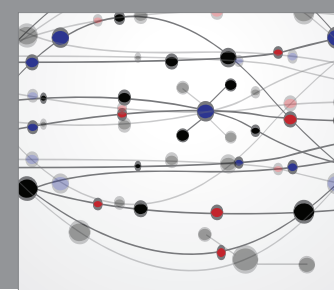

The Scientific World Journal

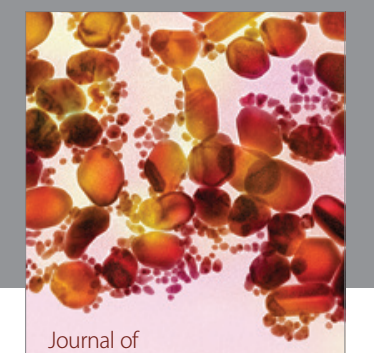

Soft Matter
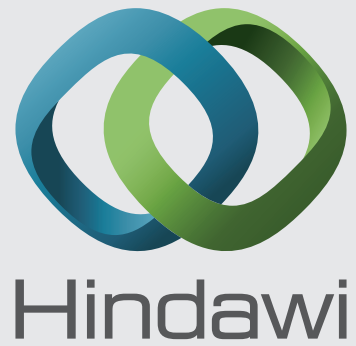

Submit your manuscripts at

http://www.hindawi.com
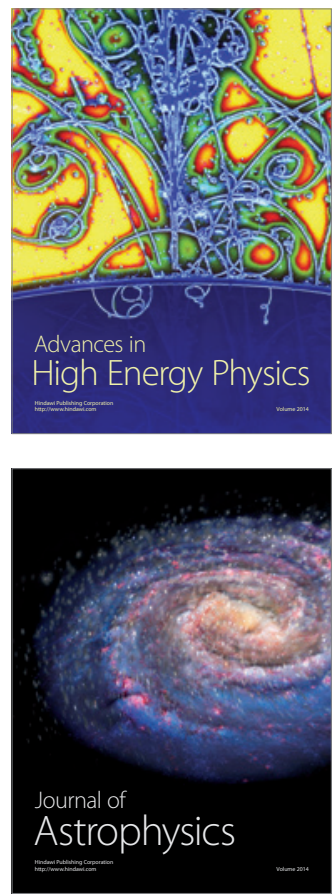
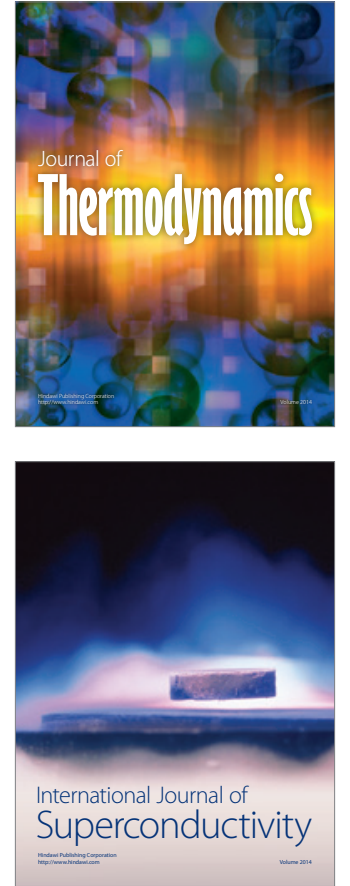
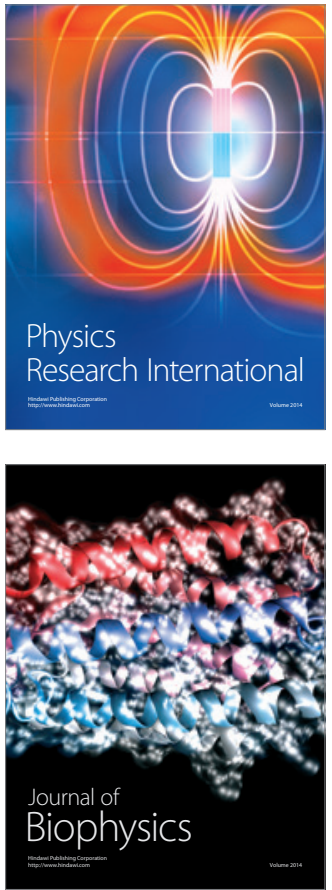
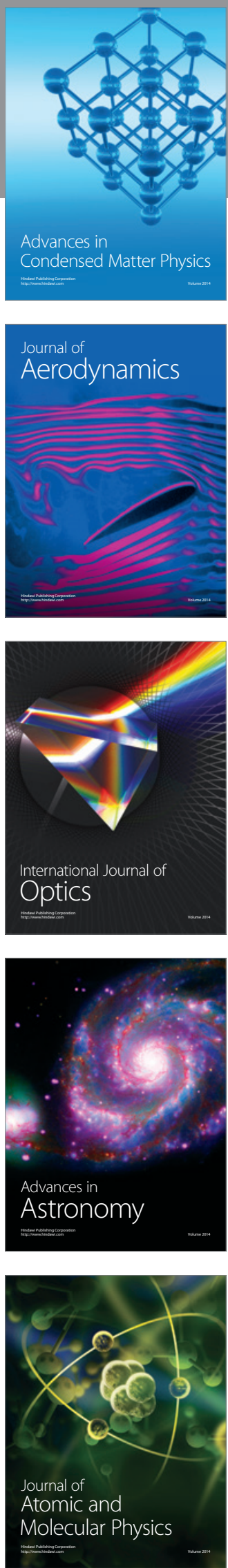